\title{
SLAMF7 Regulates Inflammatory Response In Macrophage During Polymicrobial Sepsis
}

\section{Yongjian Wu}

Department of Internal Medicine and Guangzhou Institute of Pediatrics, Guangzhou Women and Children's Medical Center, Zhongshan School of Medicine, Sun Yat-sen University, Guangzhou

Juanfeng Lao

the Fifth Affiliated Hospital, Sun Yat-sen University

\section{Miao Li}

Sun Yat-sen University School of Medicine, Shenzhen

\section{Minhao Wu}

Sun Yat-sen University

\section{Siqi Ming}

Sun Yat-sen University

\section{Lei Liu}

Shenzhen Third People's Hospital

\section{Sitang Gong}

Guangzhou Women and Children Medical Center

\section{Haibo Zhou}

The Sixth Affiliated Hospital of Guangzhou Medical University

\section{Xi Huang ( $\nabla$ huangxi6@mail.sysu.edu.cn )}

Fifth Affiliated Hospital of Sun Yat-sen University

\section{Article}

Keywords: Sepsis, SLAMF7, Inflammatory cytokines, SHIP1, TRAF6

Posted Date: February 19th, 2021

DOl: https://doi.org/10.21203/rs.3.rs-214049/v1

License: (a) (i) This work is licensed under a Creative Commons Attribution 4.0 International License. Read Full License 


\section{Abstract}

Uncontrolled microbe-triggered inflammation results in multiple organ injury and shock in sepsis. However, the regulatory mechanisms that restrict cytokine storm are still elusive. Using gene screening, we identified an immunoglobulin-like receptor called Signaling Lymphocyte Activation Molecular Family-7 (SLAMF7), as a key regulator of inflammation during sepsis. We found that the expression of SLAMF7 on monocytes and macrophages was significantly elevated in sepsis subjects and septic mice. SLAMF7 attenuated TLR dependent MAPKs and NF-KB signaling activation by co-operating with Src homology 2containing inositol-5'-phosphatase1 (SHIP1). Furthermore, SLAMF7 interacted with SHIP1 and TNF receptor associated factor 6 (TRAF6) to inhibite K63 ubiquitination of TRAF6. In addition, we found that intracellular domain tyrosine phosphorylation sites of SLAMF7 and phosphatase domain of SHIP1 were indispensable for the interaction of SLAMF7/SHIP1/TRAF6 and the modulation of cytokines production. Finally, recombinant murine SLAMF7 peptide agonist or genetic knockout of SLAMF7 in mice demonstrated that SLAMF7 confered protection against lethal sepsis and endotoxemia by suppressing inflammatory cytokines. Taken together, our findings reveal a critical negative regulatory role of SLAMF7 on cytokine storm in macrophages during polymicrobial sepsis, and therefore provide new sights into a novel diagnostic marker and therapeutic target for sepsis.

\section{Introduction}

Sepsis is among the most common causes of death in hospital, however, the pathological mechanism underlying this disease is still uncovered ${ }^{1}$. Clinical criteria for definition of sepsis remains challenging along with time ${ }^{2}$. Now, It is defined as life-threatening organ dysfunction due to a dysregulated of host immune response to infection ${ }^{3,4}$. Pathogenesis of sepsis syndrome relies critically on the activation of innate immune response by pattern recognition receptors (PRRs) in response to microbial pathogens, especially endotoxin or Gram-negative bacilli Escherichia coli (E.coli) and Pseudomonas aeruginosa $(\mathrm{PA})^{5}$. Toll like receptors (TLRs) play a critical role in the development of sepsis in response to both exogenous pathogens and endogenous ligands. Excessive inflammatory mediators, including cytokines, chemokines and growth factors released by immune cell upon TLRs activation, can lead to organ failure and even death in sepsis. A previous study demonstrates that increased TLR4 expression is correlated with mortality in the cecal ligation and puncture (CLP)-induced mouse sepsis model, whereas downregulation of TLR4 induced endotoxin tolerance ${ }^{6}$. Moreover, antagonist targeting TLR4 has been used in phase II trial that aims to decrease mortality in sepsis patients. A trend toward a lower mortality rate is observed with E5564 treatment but it still needs more investigation 7 . In these contexts, exploration of novel potential targets for modulation of inflammatory circumstance during sepsis is urgently needed.

Previous studies have reported a various of negative effector molecules induced by inflammatory stimulation, which acted as feedback loops to dampen inflammation or induce endotoxin tolerance, such as vascular endothelial growth factor receptor-3 (VEGFR-3) ${ }^{8}$, and major histocompatibility complex class II (MHC-II) ${ }^{9}$. SLAM family receptors (SFRs), a subgroup of the CD2 family of Ig-like receptors, have been 
confirmed to modulate immune cells ${ }^{10,11,12}$. Among them, SLAMF7 (also named CS1, CRACC, CD319) has been proved to inhibit TNF and IL-12p70 expression in human monocytes, whereas the specific mechanisms remain undefined ${ }^{13}$. Like other SFRs (except 2B4), SLAMF7 functions as homotypic receptors ${ }^{14}$. Upon activation, SLAMF7 in NK cells expressing EAT-2 results in activation of cellular immune responses but acts as an inhibitory receptor in the absence of EAT-2 via recruitment of a number of inhibitory phosphatases (SHP1, SHP2, SHIP1, and csk) ${ }^{15}$. Furthermore, SLAMF7 interacts with integrin Mac-1 in macrophage and utilize signals involving responsing for tumour cell phagocytosis ${ }^{16}$. Studies have focused on the functions of SLAMF7 as an important immune checkpoint in multiple myeloma immunotherapy ${ }^{17-19}$. Nonetheless, SLAMF7 may exhibit negative regulatory role on inflammation in infection disease. For example, increased SLAMF7 expression in macrophage alleviates corneal inflammation by promoting M2 polarization ${ }^{11}$. In addition, SLAMF7 negatively regulates IFN-a-mediated CXCL10 production in chronic HIV infection ${ }^{20}$. Nevertheless, its definite role and mechanism on inflammatory response in infection disease needs more elaboration.

In this study, we demonstrated that SLAMF7 (but not other SFRs) expression was significantly elevated in sepsis patients. The expression of SLAMF7 on monocytes and macrophages was correlated with clinical acute C-reactive protein (CRP) level in sepsis subjects, suggesting a new possible diagnostic biomarker for the early phase of sepsis. Consistent with that in human, SLAMF7 also acted as a "self-switch" to reduce TLR-triggered production of pro-inflammatory cytokines in macrophages in murine models of sepsis. Furthermore, SLAMF7 co-operated with SHIP1 phosphatase domain to inhibit TRAF6 autoubiquitination dependent on its phosphorylated tyrosines. Unexpectedly, SLAMF7 alone could directly interact with TRAF6 and restrict its ubiquitination independent of tyrosines. As SLAMF7 restricted TLR-dependent NF-KB and MAPKs activation, it represented a negative control mechanism of immune cells to avoid "over-reaction" during sepsis. Activation of SLAMF7 by recombinant peptide rescued mice from sepsis shock and reduced mortality. Our results, therefore, uncover the unknown function of SLAMF7 in sepsis subjects and endotoxemic mice, and may provide a roadmap as a future diagnostic marker and therapeutic target for sepsis and endotoxemia.

\section{Results}

\section{SLAMF7 expression is strongly related to sepsis .}

To evaluate the inflammatory mediators involved in TLR-signaling and the induction of endotoxin shock, we profiled the expression of cytokines, chemokines, growth factors, and receptors in peripheral blood mononuclear cells (PBMCs) from sepsis and healthy subjects by quantitative PCR. As previously shown ${ }^{8}$ 21 , our results revealed considerable induction of proinflammatory genes, including interleukin-6 (IL6) and interleukin-beta(IL 1B). In addition, multiple inflammatory-related receptors, such as TLR4 and integrin subunit alpha M (ITGAM, also called CD11B) were induced in PBMCs from sepsis patients. Among the gene screen, we analyzed the expression of SFRs, which were homotypic activated, but have not been studied in sepsis before. Interestingly, expression of SLAMF7 and SLAMF9 were significantly elevated, 
whereas SLAMF3, SLAMF4, SLAMF5, SLAMF6 and SLAMF8 were down-regulated (Fig.1a). Since cytokines from monocytes and macrophages are the major sources of inflammatory response involved in endotoxin shock or tolerance ${ }^{22,23}$, we isolated human monocytes from healthy donors, and thereafter stimulated with different doses of endotoxin (lipopolysaccharide, LPS). Focused on SFRs, we found that expression of SLAMF2, SLAMF7, SLAMF8 and SLAMF9 were all elevated, but SLAMF7 was more endotoxin- dose-dependent (Fig. 1b). Data suggested that SLAMF7 expression was endotoxin inducible, consistent with the report that SLAMF7 expression elevated on monocytes after TLR ligands stimulation $^{13}$.

Furthermore, we confirmed that the percentage of SLAMF7 in CD11 $\mathrm{b}^{+}$myeloid cells but not CD $3^{+} \mathrm{T}$ cells was significantly increased in sepsis subjects ( $n=83$, Supplementary Table 1$)$ compared to healthy donors ( $n=81$, Supplementary Table 1 ) by flow cytometry (Fig. 1c,d). Then we investigated whether expression of SLAMF7 in sepsis exhibited disease specificity. As a result, we found that SLAMF7 expression was not related to gender, age, infectious microbe and different disease types (Supplementary Fig. 1a-d). Interestingly, data showed that $S L A M F 7^{+} C D 11 b^{+}$cells percentage was strongly correlated with the C-reactive protein (CRP) concentration ( $r=0.56$ ) (Fig. 1e). Considering that CRP is a marker of disease prognosis ${ }^{24}$, we evaluated the expression of SLAMF7 during sepsis therapy. We found that levels of CRP (Fig. 1f) and percentage of SLAMF ${ }^{+} C D 11 b^{+}$cells (Fig. 1g) were both gradually decreased from pretreatment to day 7 post-therapy. Overall, our results showed that expression of SLAMF7 was associated with sepsis process, suggesting a possible diagnosis and prognosis marker of SLAMF7 for sepsis.

\section{SLAMF7 expression is induced by TLR/NF-KB signaling in macrophages.}

Upon recognition of LPS or bacteria, TLR4 initiates MyD88-dependent pathway for NF-KB activation, which has been linked to an excessive inflammation and endotoxin shock ${ }^{25}$. Therefore, we sought to examine whether TLR-MyD88-NF-KB signaling activation contributes to SLAMF7 expression. Using different TLR ligands to stimulate RAW264.7 cells, we observed that LPS induced the most Slamf7 expression than other ligands (Pam3Csk4 for TLR1/2, LPS for TLR4, R848 for TLR7/8, poly(I:C) for TLR3) (Fig. 2a), suggesting that TLR4 engagement was indispensable. To avoid discrimination, we confirmed the expression of Slamf7 in BMDM and huaman monocytes in response to LPS stimulation (Supplementary Figure 2a). Data showed that both TLR4 ligand stimulation and PA infection induced Slamf7 expression of RAW264.7 cells in a time and dose dependent manner (Fig. $\mathbf{2 b , c}$ and Supplementary Figure $2 b, c$ ). As MyD88 is known to signal downstream of TLR4 ${ }^{26}$, we investigate whether it regulates the transcription of Slamf7. After knockdown of MyD88, we found that Slamf7 expression was inhibited in response to LPS stimulation for 24h and 48h (Supplementary Fig. 2d,e). Sequence analysis with the JASPAR program predicted several potential NF-KB and AP-1 binding sites in the promoter region of Slamf7 (Supplementary Fig. 2f). The transcriptional level of Slamf7 was almost diminished by IKB/IKK pharmacological inhibitor (BMS345541) followed LPS stimulation at $24 \mathrm{~h}$ or $48 \mathrm{~h} \nabla$ while MAPKs inhibitors (led to AP-1 activation) had minimal influence (Supplementary Fig. 2g). Consistant with that, another chemical inhibitor JSH-23 targeting translocation of p65 subunit of NF-KB, 
also decreased Slamf7transcriptional levels both in BMDM and RAW264.7 cells (Supplementary Fig. $2 \mathrm{~h}, \mathbf{i})$. As previous study reported ${ }^{13}$, these data suggested that TLR-MyD88-NF-KB signaling promoted SLAMF7 expression during endotoxin process.

\section{SLAMF7 regulates proinflammatory cytokine production in macrophages.}

Early death in sepsis is largely due to a cytokine storm and the associated multiorgan failure ${ }^{27}$. It is well known that macrophages are the main mediators of TLR-triggered innate inflammatory responses ${ }^{11}$, then we investigate the role of SLAMF7 in macrophages. Firstly, we constructed cell line steadily expressing SLAMF7 by lentivirus transfection (RAW-SLAMF7) (Supplementary Fig. 3a). Overexpression of SLAMF7 successfully reduced pro-inflammatory cytokines (Tnf, //1b and $/ / 6$ ) levels (Fig. 2d and Supplementary Fig. 3b). Consistent with that, BMDM treated with rmSLAMF7 also decreased expression levels of cytokines (Fig. 2e). On the other hand, we observed excessive pro-inflammatory cytokine production in SLAMF7 KO BMDM compared with WT BMDM (Fig. 2f). Consistently, IL-1b and IL-6 production were up-regulated after SLAMF7 was knocked out by crispr-cas 9 technique (Fig. $\mathbf{2 g}$ and Supplementary Fig. $3 \mathrm{c}$ ). Taken together, these data indicated that SLAMF7 exerted an inhibitory effect on TLR4-triggered inflammatory response in macrophages.

\section{SLAMF7 suppresses TLR4-triggered inflammatory signaling pathway by activating phosphorylation of SHIP1.}

As TLR4-triggered phosphoinositide 3-kinase and protein kinase AKT (PI3K/AKT), NF-KB and MAPKs activation could lead to large amount of inflammatory cytokines in sepsis, we next investigated whether SLAMF7 affected these signaling pathways. We observed that overexpression of SLAMF7 inhibited the phosphorylation of Akt (Fig. 3a), ERK, p38 (Fig. 3b) and IKKa/ $\beta$ (Fig. 3c), but had minimal influence on JNK (Fig. 3a-c). On the contrary, NF-KB (IKKa/ $\beta$ ) and MAPKs signaling ( $\mathrm{p}$-ERK, p-JNK, p-p38) were activated when SLAMF7 was knocked out in BMDM or knocked down in RAW264.7 cells after LPS stimulation (Fig. 3d and Supplementary Fig. 4a). Previous studies demonstrate that IKKa/ $\beta$ (IkB kinase) phosphorylates IkB lead to its dissociation from NF-KB, thereby allowing NF-KB to enter the nucleus ${ }^{31}$. We found that the amount of p65 translocation from cytoplasma to nuclei decreased in SLAMF7 overexpressing cells (Fig. 3e), but increased in SLAMF7 KO BMDM (Fig. 3f). A previous study reports that AKT signaling pathway functions in inflammatory process by regulating NF-KB Ser phosphorylation, p38 and ERK activation ${ }^{28}$. We therefore assessed the role of SLAMF7 on cytokine production by inhibiting AKT or NF-kB using Ly294002 (Fig. 3g) or JSH23 (Fig. 3h) respectively. We observed that these two inhibitors abrogated the decrease of pro-inflammatory cytokine expression in RAW-SLAMF7 cells (Fig. 3g,h). These results indicated that SLAMF7 inhibited inflammatory response by disturbing AKT signaling pathway. To further elucidate how SLAMF7 mediated these effects, we next investigated the adaptor for downstream signal. It has been reported that SFRs utilize SLAM-assocated-adaptor-proteins (SAPs) to transduce signaling from membrane into cytoplasma, including EAT-2 and SAP ${ }^{29}$. Nevertheless, previous study reported that EAT-2 expressed faintly in primary human monocytes ${ }^{13}$, suggesting other novel adaptors for SFRs molecular. Here, we used rmSLAMF7 to activate SLAMF7 in BMDM to detect candidate adaptors 
molecule expression. Interestingly, the Eat-2 and Sap expression had no difference after rmSLAMF7 stimulation, but SHIP1 mRNA expression increased about 3 folds (Fig. 3i). To ascertain this observation, we investigated the phosphorylation of SHIP1 ( $p$-SHIP1) in RAW-SLAMF7 cells or SLAMF7 KO BMDM, followed by LPS stimulation for different time. Data showed that RAW-SLAMF7 increased the phosphorylation of SHIP1 (Fig. 3j and Supplementary Fig.4b). Contrarily, SLAMF7 knockout attenuated the level of p-SHIP1 (Fig. 3k). Accordingly, SHIP1 knockdown also disturbed SLAMF7 signaling transduction. Transfection with siRNA targeting SHIP1 in RAW264.7 (Supplementary Fig. 4c) significantly reduced p-SHIP1 expression, and the inhibition of cytokine production by over-expression of SLAMF7 was reversed (Fig. 3l). A recent study by Guo et al showed that SLAMF7 interacts with SHIP1 and CD45 in multiple myeloma cells dependent of Src kinase ${ }^{30}$. To elucidate how SLAMF7 mediated these effects in macrophages, we analyzed the contribution of various signaling kinases in SLAMF7-dependent cytokine inhibitions. Results showed that pharmacological inhibitors targeting TBK1 abrogated the inhibition function on inflammatory cytokine responses in RAW264.7 cells, but other kinases, namely Src and Syk were not involved in SLAMF7 signaling pathway (Supplementary Fig. 4d,e), indicating that SLAMF7 functioned in macrophages was different from other cell types. Together, these data suggested that SHIP1 activation was involved in SLAMF7 signaling in suppressing TLR4-triggered inflammatory responses.

\section{SLAMF7 suppresses TRAF6 Ubiquitination by Co-operation with SHIP1.}

Previous studies reported that SLAMF7 can interact with SHIP1 to mediate inhibitory effects in the absence of activated adaptor EAT-2 in NK cells ${ }^{15,31}$. To investigate whether SLAMF7 can interact with SHIP1 directly, we transfected Flag-tagged SLAMF7 and HA-tagged SHIP1 in 293T cells. Consistant with prior studies ${ }^{15}$, we found that SLAMF7 interacted with SHIP1 directly (Fig. 4a,b). TNF receptorassociated factors (TRAFs) play a central role in the TNF-a, IL-1- $\beta$, and LPS-induced signaling pathways $^{32}$. Binding of LPS to TLR4 (Toll-like receptor 4) triggers the recruitment of MyD88 and IRAK1/4, which then recruits TRAF6 and triggers downstream signaling ${ }^{33}$. Here, we observed that activating SLAMF7 obviously up-regulated TRAF6 expression in LPS-stimulated BMDM (Supplementary Fig. 5). Endogenous immunoprecipitation ananlysis showed that SLAMF7 interacted with TRAF6 other than SHIP1, which was dependent on the SLAMF7 expression after LPS stimulation (Fig. 4c). Exogenous coprecipitation showed the direct interaction of SLAMF7 and TRAF6 (Fig. 4d), as well as SHIP1 and TRAF6 (Fig. 4e). Furthermore, we confirmed that SLAMF7 co-localized with SHIP1 and TRAF6 by laser confocal fluorescence microscopy (Fig. 4f). In line with the endogenous immunoprecipitation, data suggested that SLAMF7 interacted with SHIP1 and TRAF6 directly.

A previous study has demonstrated that SHIP1 reduces TRAF6 autoubiquitination in NK cells ${ }^{34}$. Here, we found HA-tagged ubiquitination (Ubs-HA) of Flag-tagged TRAF6 was reduced by transfection of SHIP1 exactly in a dose dependent manner (Fig. $\mathbf{4 g}$ ). Unexpectedly, we found that SLAMF7 also reduced TRAF6ubiquitination independent of SHIP1, but the inhibitory function was enhanced by co-operation with SHIP1 (Fig. 4h). TRAF6 is a RING domain protein, which functions to catalyze the synthesis of unique 
polyubiquitin chains linked through lysine-48 (K48) and lysine-63 (K63) of ubiquitin, and K63 is essential for the activation of IKK and AKT signaling pathway ${ }^{35,36}$. We therefore sought to investigate the type of ubiquitination of TRAF6 regulated by SLAMF7 and SHIP1. Through lysine mutation of ubiquitin, we observed that SLAMF7 co-operated with SHIP1 to attenuate K63 linked autoubiquitin but not K48 linked (Fig. 4i). Moreover, we found that the total ubiquitination increased after knockdown of SLAMF7, and increase accumulated by using proteasome inhibitor (MG132), indicating that SLAMF7-induced ubiquitination decrease was mediated independently by proteasome degradation mechanism (Fig. 4j). Finally, we found that $T n f, I L 1 b$ and $/ 16$ expression inhibited by SLAMF7 were reversed after transfection with TRAF6 plasmid, indicating that TRAF6 affected SLAMF7 signaling in regulating cytokine production (Fig. 4k). Collectively, the results revealed that SLAMF7 interacted with TRAF6 and restricted its K63 autoubiquitination by co-operation with SHIP1 to regulate inflammatory cytokines production.

\section{The interaction of SLAMF7, TRAF6 and SHIP1 is dependent on phosphatase domain of SHIP1.}

Next, we decided to determine which domains of SLAMF7 and SHIP1 were required for the interaction and suppression of TLR4-triggered inflammatory responses. SLAM family molecules contain a N-terminal extracellular domain, a single transmembrane domain and a cytoplasmic tail ${ }^{37}$. Unlike the other members, in mice, SLAMF7 has a relatively long tail and three tyrosine-phosphorylation sites in the cytoplasmic domain (Y261, Y266 and Y281) ${ }^{38}$ (Fig. 5a). SHIP1 possesses an amino-terminal Src homology $2(\mathrm{SH} 2)$ domain that binds preferentially to the sequence $\mathrm{PY}(\mathrm{Y} / \mathrm{S} / \mathrm{T}) \mathrm{L}(\mathrm{M} / \mathrm{L})$ and has been shown to bind to the tyrosine phosphorylated forms of Shc (SH2 domain), a centrally located phosphoinositol phosphatase domain that selectively hydrolyzes the 5'-phosphate (EEP domain), and a critical proline rich C-terminus that binds a subset of SH3-containing proteins (P rich domain) ${ }^{39,40}$ (Fig. 5b). Firstly, we generated a panel of serial-deletion constructs of SLAMF7 (deletion of intracellular, transmembrane and extracellular domain) and performed coimmunoprecipitation assays. Results showed that deletion of any domain of SLAMF7 failed to bind SHIP1, which indicated the interaction was dependent on full-length SLAMF7 (Fig. 5c). However, deletion of SLAMF7 intracellular domain was unable to bind TRAF6 compared to other two truncated fragments and full-length SLAMF7, which demonstrated the interaction between SLAMF7 and TRAF6 was dependent on intracellular domain of SLAMF7 (Fig. 5d). In addition, we ascertained that EEP domain of SHIP1 was responsible for the interaction with SLAMF7 (Fig. 5e) or TRAF6 (Fig. 5f). Moreover, EEP domain of SHIP1 assisted SLAMF7 to inhibit autoubiquitination of TRAF6 significantly (Fig. 5g). However, SHIP1 without EEP domain only partially reversed the inhibition, which suggested that SLAMF7 also had impact on TRAF6 autoubiquitination independent of EEP domain (Fig. 5g).

To elucidate whether SLAMF7 tyrosines mediated the signal transduction, we expressed SLAMF7 with three intra-cytoplasmic tyrosines $(Y)$ mutated to phenylalanines $(F)$ ( $Y$ to $F$ mutations) (Fig. 5a). From the co-immunoprecipitation of mutated SLAMF7 with SHIP1, we found that SLAMF7 with Y281 mutation failed to bind SHIP1, indicating that Y281 of SLAMF7 played a decisive role on the interaction (Fig. $5 \mathbf{h}$ ). Furthermore, we showed for the first time that the interaction between SLAMF7 and TRAF6 was independent of tyrosines (Fig. 5i). These results suggested that SHIP1 and TRAF6 showed 
no competitive binding to SLAMF7. Lastly, we investigated whether the tyrosine mutations in SLAMF7 resulted in impaired cytokines production. Then RAW264.7 cells were transfected with three mutations of SLAMF7 to detect cytokine production respectively. Intriguingly, SLAMF7 with tyrosine mutations failed to down-regulate cytokine expression, including $T N F, \| 1 b$ and $/ / 6$ (Fig. 5j). Among the three tyrosine mutations, Y281 mutation increased the cytokine production most significantly (Fig. 5j). Overall, our results confirmed that the tyrosines of SLAMF7 is crucial for cytokine production.

\section{Activation of SLAMF7 rescues septic mice by inhibiting inflammatory response}

To further investigate the role of SLAMF7 in sepsis, WT C57BL/ 6 mice were subjected to sepsis by LPS(endotoxemia model) stimulation, PA囚bacterial infection model) infection and CLP (polymicrobial sepsis model) ${ }^{41}$. Consistent with data from peripheral blood of sepsis patients, the percentage of $\mathrm{SLAMF7}^{+} \mathrm{F} 4 / 80^{+}$macrophages was elevated significantly after LPS stimulation , PA infection or CLP in peritoneal lavage (Fig. 6a). Data indicated that SLAMF7 expression in macrophages was upregulated during endotoxin stimulation and bacterial infection in mice. To confirm the role of SLAMF7 in the pathogenesis of sepsis, we observed the survival and histopathology of mice after sepsis induction. Since SLAMF7 can be activated homotypically ${ }^{42}$, we utilized recombinant mouse SLAMF7 peptide (rmSLAMF7, R\&D) targeting extracellular domain to investigate the its function in vivo. Firstly, WT mice were subjected to LPS , PA and CLP surgery to induce sepsis, and mortality was monitored. We found that rmSLAMF7 treatment obviously improved the survival of septic mice (Fig. 6b-d). Thus, SLAMF7 receptor appeared to play an important role on rescuing septic mice. Moreover, mice treated with rmSLAMF7 presented less tissue injury and leukocytic infiltrationfter with sepsis (Fig. $\mathbf{5 e}$ and Supplementary Fig. 6a-c). Together, these data indicated that SLAMF7 activation rescues the survival and pathology of septic mice. To investigate whether SLAMF7 regulated the inflammation induced by sepsis in vivo, enzyme-linked-immunosorbent assay (ELISA) was used to determine the concentrations of key inflammation factors in the supernatant of liver, lung ,PL and serum of mice. We observed significant reductions in the expression levels of the proinflammatory cytokines TNF-a, IL-1 $\beta \square$ and IL-6 in the rmSLAMF7 treatment group with CLP- and LPS-induced sepsis (Fig. $6 \mathrm{f}$ and Supplementary Fig. 7a). These results suggested that SLAMF7 alleviated LPS- or CLP- induced inflammation and protected septic mice against the acute inflammatory process. Furthermore, we found that the percentage of $\mathrm{F} 4 / 80+$ macrophages from the PL had no difference in WT mice treating with rmSLAMF7 compared with control (Fig. 6g), but expression levels of TNF-a, IL-1 $\beta$ and IL-6 were decreased in rmSLAMF7-treated group after CLP for $24 \mathrm{~h}$ (Fig. $6 \mathrm{~h}$ ). Thus, SLAMF7 attenued inflammatory response by regulating the homeostasis of macrophages in sepsis.

\section{Knockout of SLAMF7 contributes to the development of exacerbated inflammatory response in sepsis}

To further confirm the function of SLAMF7 on the development of sepsis, WT and SLAMF7-deficient (SLAMF7 KO) mice were subjected to LPS , PA and CLP surgery to induce sepsis, and mortality was monitored. We found that the mortality of SLAMF7 KO is higher compared with that of WT mice (Fig. 7ac). In addition, more severe infiltration of polymorphonuclear cells and interstitial pneumonitits were 
found in SLAMF7 KO mice after CLP compared to WT mice (Fig. 7d). These data suggested that SLAMF7 was vital in improving survival and reducing pathological damage in septic mice. Furthermore, We demonstrated that SLAMF7 KO mice displayed a remarkable increase levels of inflammatory cytokines (TNF-a, IL-1 $\beta$ and IL-6) in comparison with WT mice post-CLP (Fig. 7e) or LPS treatment (Supplementary Fig. 7b). These results indicated that SLAMF7 was required for inhibition of excessive inflammation in sepsis. Consistant with that, SLAMF7 knockout didn't affect the percentage of macrophages (Fig. 7f), but more secreted cytokines were found in SLAMF7 KO mice after CLP (Fig. 7g). Taken together,our results indicated that SLAMF7 attenuated sepsis-induced mortality and lung injury through suppressing excessive inflammatory response.

\section{Discussion}

SLAMF7 is an immunoglobulin superfamily receptor which plays a key role on NK cell cytolytic activity in tumor $^{43}$. In this study, we demonstrated that SLAMF7 was inducibly expressed on macrophage in response to TLR ligands and bacterial infection, and interacted with SHIP1/TRAF6 to attenuate MAPKs and NF-KB signaling and pro-inflammatory cytokines production. Finally, recombinant murine SLAMF7 peptide agonist or genetic knockout of SLAMF7 in mice demonstrated that SLAMF7 confered protection against lethal sepsis and endotoxemia by suppressing inflammatory cytokines. To the best of our knowledge, this study explored a negative regulatory role of SLAMF7 on macrophage, which provide new sights into a novel therapeutic target for sepsis.

Unlike various intracellular negative molecules, SFRs are surface receptors and possess tyrosine kinase activity to recruit effectors. The unique property of SLAMF7 has been employed for clinical applications, including usage of anti-SLAMF7 antibodies or SLAMF7-directed chimeric antigen receptor (CAR) modified T cells for tumor therapy ${ }^{44-46}$. In 2013, PDL241, a novel humanized monoclonal antibody of SLAMF7, inhibited IgM production from plasmablasts and plasma cells, showing beneficial effect on joint related parameters ${ }^{47}$. Here we first provided evidence that SLAMF7 expression was significantly elevated in patients with sepsis and decreased during therapy. SLAMF7 expression on myeloid cells showed an obvious correlation with clinical CRP concentration in the serum of patients, which suggested a new diagnostic biomarker for sepsis ${ }^{21}$. Aside from the phagocytosis function, regulation on macrophage polarization and bacterial infection ${ }^{11,38}$, we identified that SLAMF7 expressing on macrophages modulated cytokines production by inhibiting AKT, NF-KB and MAPKs signaling pathways. Furthermore, triggering SLAMF7 signaling by recombined peptide rescued mice from lethal sepsis, contributing to better overall survival.

Previous studies have documented that multiple members of SFRs participate in infectious diseases ${ }^{48-50}$. Evidence for direct interaction of SLAMF1 with E. coli outer membrane porins C (OmpC) and OmpF is shown in a cell-based luciferase reporter assay ${ }^{51}$, while SLAMF2 is implicated in the recognition of nonopsonized E. coli via caveolae, resulting in phagocytosis by mast cells ${ }^{52}$. Moreover, SLAMF1 also engages with Measles virus protein $\mathrm{MV}-\mathrm{H}$, which provides templates for antiviral drug design ${ }^{53}$. Different 
with other SLAMFs, SLAMF7 is a self-ligand and homophilic interaction of its self regulates NK cell cytolytic activity in tumor ${ }^{54}$. To date, the definite function and mechanism of SLAMF7 in infectious disease are still unclear. It has previously been reported that increased SLAMF7 expression in macrophage alleviates corneal inflammation caused by pseudomonas aeruginosa ${ }^{11}$. In addition, SLAMF7 negatively regulates IFN-a-mediated CXCL10 production in chronic HIV infection ${ }^{20}$. In this study, we found that the expression of SLAMF7 in CD11 $\mathrm{b}^{+}$subsets had no obvious relation with infectious microbe types in sepsis patients. A previous report revealed that adherent or LPS-activated monocytes expressed SLAMF7 via NF-KB and PI3K pathway. Cross-linking SLAMF7 reduced secretion of TNF and IL12 p70 by LPS-activated monocytes ${ }^{13}$. In our study, we analyzed the SLAMF7 promoter, and found that NF-KB but not AP-1 regulated SLAMF7 transcriptional levels in murine macrophages. Importantly, SLAMF7 could reduce inflammatory cytokines (TNF-a, IL-1 $\beta$, IL-6) secretion in vivo and in vitro, indicating that SLAMF7 could protect against sepsis by inhibiting cytokine storm. Extensive research on the wiring of signal transduction pathways has uncovered a level of complexity dictating of PI3K/AKT pathways. One mechanism involved in the signaling transduction pathways has been attributed to the direct phosphorylation of the amino acid residue T23 on IKKab by AKT, thereby leading to activation of this kinase upstream of NF-kB ${ }^{55}, 56$. However, other studies found that AKT blocked ERK signaling through inhibition of C-Raf on T25957, 58. In our results, knockdown of SLAMF7 in BMDM promoted the activation of AKT and NF-KB pathways and increased phosphorylation of ERK, JNK and p38. Blockade of PI3K/AKT and NF-kB pathways by inhibitor abolished the impairment of cytokines production by SLAMF7, which indicated that SLAMF7 exerted its biological functions via the inhibition of these two signaling pathways.

Previous studies show that SLAMF7 positively regulates NK cell function dependent on the adaptor EAT2, but not related adaptor SAP. In the absence of EAT-2, SLAMF7 potently inhibits NK cell function by recruiting inhibitory effectors of SHP-1, SHP-2, SHIP1, Csk and Fyn ${ }^{15}$. In macrophage, SLAMF7 interacts with integrin Mac-1 and utilize signals involving responsing for tumour cell phagocytosis ${ }^{16}$. Thus, SLAMF7 exerts activating or inhibitory influences on cells of the immune system depending on the cellular context and the availability of effector proteins. Notably, the functions of SLAMF7 and its adaptors in cytokine modulation process in other cell types has not been explored. Our results showed that the phosphorylation of SHIP1 decreased in BMDM knockdown with SLAMF7 siRNAs or knockout, and the pro-inflammatory cytokines downregulated by SLAMF7 were reversed by knockdown of SHIP1. Furthermore, we found that SLAMF7 directly interacted with SHIP1 in murine macrophages. Supporting with our findings, SHIP1 and SLAMF7 interacts in multiple myeloma cells depending on Src kinase, and Elotuzumab (a humanized monoclonal antibody of SLAMF7 mentioned before) triggers prominent tyrosine phosphorylation of SHIP1 in IM-9 cells ${ }^{30}$. However, we failed to detect the alteration of proinflammatory cytokines (Tnf and I/6) by Src kinase treatment in ovexpressing SLAMF7 cell responsing to LPS stimulation. However, other kinase ,TBK1 participated in regulating cytokines production.

SHIP1 is important in endotoxin tolerance and LPS-induced cytokine production restriction ${ }^{59}$. SHIP1 possesses an amino-terminal $\mathrm{SH} 2$ domain that binds preferentially to the sequence $p Y(Y / S / T) L(M / L)$, a centrally located EEP domain that selectively hydrolyzes the 5'-phosphate, and a P rich domain that binds 
a subset of SH3-containing proteins ${ }^{40}$. Although SHIP1-mediated inhibition of TLR4 induced-PI3K activation is dependent on its phosphatase activity ${ }^{60}$, An et al found that phosphatase activity-disrupted mutant SHIP1 (P671, D675 and R676 sites in phosphatase activity domain) remains inhibitory to LPSinduced TNF production ${ }^{60}$. We here claimed that phosphatase domain of SHIP1 mediated the interaction with SLAMF7, and assisted to inhibit autoubiquitination of TRAF6. It has been reported that SLAMF7 has unique introcytoplasmic domain distinct from other members in SFRs, which is not the typical binding sequence for SAP61. Our data showed that the interaction between SLAMF7 and SHIP1 had been impaired after knockout of EEP domain but not $\mathrm{SH} 2$ or $\mathrm{P}$ rich domain. Thus we supposed that the EEP domain had a essential role in SLAMF7 and SHIP1 interaction upon TLRs signaling. Guo et a/ have reported that the long transcript of SLAMF7 (SLAMF7-L) in multiple myeloma cells, containing tyrosines binds to SHIP1 rather than short transcript of SLAMF7 (SLAMF7-S) ${ }^{30}$, consistent with that, our results showed that SLAMF7 lacking of any function domains losed the ability to interact with SHIP1. Moreover, the mutation with the indispensable tyrosines of SLAMF7 (Y261, Y266, Y281) has been reported before ${ }^{38}$. Although these tyrosines were couple to SAP or EAT-2 in multiple myeloma cells ${ }^{30}$, we discovered the interaction of SLAMF7 with SHIP1 was lost with Y281 mutation and decreased with Y261 and Y281 mutation in macrophages.

Ubiquitin modification of signaling proteins through K48-linked ubiquitin chains typically promotes their degradation by proteosome-dependent mechanisms. In contrast, ubiquitin modifications through non-K48 linkages, such as $\mathrm{K} 63$ ubiquitin linkages, can activate multiple signaling pathways ${ }^{35}$. Recently, a large number of negative regulators have been reported to suppress autoubiquitination of TRAF6, where the deubiquitinating enzyme $A 20$ is required for termination of TLR activity by removing ubiquitin from

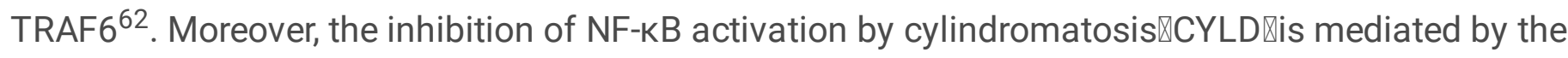
deubiquitination and inactivation of TNFR-associated factor 2 (TRAF2) and TRAF6, which downregulates the cytokine secretion ${ }^{63}$. The heat-shock-protein (HSP70) inhibits LPS-induced NF-KB activation by binding TRAF6 and preventing its ubiquitination ${ }^{64}$. Kim et al have discovered that inositol polyphosphate multikinase (IPMK) noncatalytically enhances TLR signaling by stabilizing TRAF6 in macrophages. IPMK depletion blunts TLR-mediated signaling and proinflammatory cytokines secrection, rendering mice resistant to septic responses ${ }^{65}$. Our results showed that SLAMF7 inhibited TRAF6 K63 ubiquitination by co-operating with SHIP1, and interacted with TRAF6 independent of three tyrosine sites. Knockdown of SLAMF7 increased overall ubiquitination in BMDM independent of proteasome pathway, suggesting that SLAMF7 had no obvious influence on the proteasome-degradation. Li and colleagues ${ }^{34}$ found that arrestin 2 associated with phosphorylated TIGIT for further recruitment of SHIP1 to impair the TRAF6 autoubiquitination, leading to suppression of IFN production in NK cells. Consistent with that, we innovatively discovered that EEP domain of SHIP1 facilitated dramatically the inhibition of ubiquitination of TRAF6. However, the inhibition is recovered when the EEP domain of SHIP1 is lacking. Bao and colleagues ${ }^{39}$ claims that the adaptor CD2AP/SHIP1 complex associates with Cbl, and inhibits its E3 ubiquitin ligase activity in plasmacytoid dendritic cells upon BDCA2 cross-linking. The first $\mathrm{SH} 3$ domain of CD2AP binds to the P-rich domain of SHIP1. As shown in our results, SLAMF7 interacted with SHIP1 by 
EEP domain, and the P-rich domain was available for interaction with other molecules; we hypothesize that undetectable SH3 containing molecules may associate with SHIP1 in endotoxin process. Here, we clearly demonstrated that SLAMF7 recruited SHIP1 and TRAF6 to inhibit pro-inflammatory cytokine productions.

In summary, this study provides the first evidence that SLAMF7 is an essential molecular switch for the regulation of pathological cytokine productions during sepsis. The role of SLAMF7 on inflammatory cytokines is dependent on ubiquitination of TRAF6 by co-operation with SHIP1. Our study sheds light on the interaction between SLAMF7 and TRAF6/SHIP1, and may provide a roadmap in development of novel diagnostic marker and therapeutic target for polymicrobial sepsis and endotoxemia.

\section{Method}

\section{Ethics statement}

All experimental protocols were approved by Sun Yat-sen University. The methods used in this study were carried out in accordance with the approved guidelines. All animal experiments were approved by the Animal Ethics Committee of the Fifth Affiliated Hospital, Sun Yat-sen University, and performed in accordance with the guidelines of Animal Care and Use of Sun Yat-sen University. The human cohort study was approved by the following institutional review boards: Guangzhou Women and Children's Hospital and Sun Yat-sen University prior to the commencement of the study. All participants provided a written informed consent for their participation in the study (patients were identified following sepsis definitions according to international guidelines) (Supplementary Table 1).

\section{Mice}

WT C57BL/6 mice were purchased from Sun Yet-sen University Animal Supply Center. SLAMF7 KO mice in the C57BL/ 6 background were obtained from the Nanjing Biomedical Research Institute of Nanjing University. All animal experiments were performed in accordance with the National Institutes of Health Guide for the Care and Use of Laboratory Animals, with the approval of the Scientific Investigation Board of Sun Yat-sen University (Guangdong, China).

\section{Establishment of sepsis model}

The endotoxin shock mouse model was established by intraperitoneal injecting mice with LPS (L2880, Sigma) (non lethal: $20 \mathrm{mg} / \mathrm{kg}$;lethal:40 mg/kg). Polymicrobial sepsis was induced using the cecal ligation and puncture (CLP) method as described previously. Briefly, mice were anesthetized with chloral hydrate injection of $0.4 \mathrm{~g} / \mathrm{kg}$. A small midline incision was made to expose the cecum through the skin and peritoneum under aseptic conditions. Approximately $75 \%$ of the cecal appendage was ligated midway between the cecal base and the distal pole, using $4 / 0$ surgical silk. Then, double punctures were made using an 18-gauge needle expelling a small amount of feces into the abdominal cavity. The cecum was then returned to the peritoneal cavity and the incision was closed using two layers of sutures. 
For bacterial infection, Pseudomonas aeruginosa (PA, standard strain 19660) in mid-logarithmic growth were collected, counted on agar plates and then resuspended in $0.9 \% \mathrm{NaCl}$. The concentration of PA to infect mice is $4 \times 10^{7}$ colony-forming unit per kg body weight. Recombined peptide of mouse SLAMF7 (4628-SF) was injected intraperitoneally. Mouse serum, peritoneal lavage and organ grinding fluid were collected and cytokine production including TNF-a, IL-1b and IL- 6 were measured by murine ELISA Kit (R\&D System, Minncapolis, MN) according to the manufacturers' instructions.

\section{Flow cytometry assay}

Single-cell suspensions were counted and collected. For surface staining, cells were stained for CD11b (ICRF44), CD3 (UCHT1) and SLAMF7 (162.1) for the clinical samples, CD11b (clone M1/70), CD3 (clone 145-2C), NK1.1 (clone PK136) and SLAMF7 (4G2) for mouse samples in PBS containing 1\% BSA for 20 min on ice in the dark and then washed. Dead cells were excluded using viability dye 506 or 660 , then washed three times with FACS buffer and fixed in a solution of $1 \%$ paraformaldehyde (BBI Life Science, Shanghai, China). In some cases, sing-cell suspensions were stimulated with LPS (L4524, Sigma) in combination with brefeldin A (GolgiPlug, BD Biosciences). After 6 h, cells were surface stained for antibodies then washed twice with FACS buffer stained with fluorochrome-conjugated antibodies against IL-6 (MQ2-13A5), IL-10 (554707), TNF (MAB11), p-AKT (SDRNR), or their appropriate isotype controls rat IgG1, rat IgG2b for 40min in fixation/permeabilization buffer set (eBioscience, San Diega, CA). Mouse single-cell suspensions were stained with fluorochrome-conjugated antibodies against surface markers. All antibodies were purchased from BD Biosciences, eBioscience or Biolegend (CA, USA) except where otherwise indicated. All the flow cytometry assays were performed on FACS Canto II (BD, NJ, USA), and data were analyzed by Flowjo software.

\section{Cell culture and inhibitor reagents}

Murine macrophage-like RAW264.7 cells (ATCC TIB-71), 293T (ATCC CRL-11268) and 293FT (ATCC PTA5077) cells were cultured in DMEM supplemented with $10 \%$ fetal bovine serum and $100 \mathrm{U} / \mathrm{ml}$ penicillin, $100 \mu \mathrm{g} / \mathrm{ml}$ streptomycin at $37^{\circ} \mathrm{C}$ with an atmosphere of $5 \% \mathrm{CO} 2$. NF-kB inhibitor (JSH-23), ERK inhibitor (U0126, Sigma), JNK inhibitor (SP600125), p38 MAPK inhibitor (SB203580), AKT inhibitor (Ly294002), TBK1 inhibitor (BX-795), Src inhibitor (PP2), Btk inhibitor (Ibrutinib) and Syk inhibitor (R406) were obtained from Selleck Chemicals (Houston, TX) except were indicated otherwise. TLR1/2 ligands (Pam3Csk4), TLR7/8 ligands (R848) and TLR3 ligands (poly(l:C)) were obtained from InvivoGen (CA, USA).

\section{Plasmid construction and transient transfection}

The cDNA sequence of murine TRAF6, SLAMF7 and SHIP1 with an N-terminal tag were amplified by reverse transcription-PCR and cloned into pcDNA3.1(+) vector following the manufacturer's protocol. The SLAMF7- $\Delta$ extra, SLAMF7- $\Delta$ trans and SLAMF7- $\Delta$ intra were generated by deleting extracellular domain, the trans-membrane domain and the intracellular domain. The tyrosine mutations of SLAMF7 at Y261, Y266 and Y281 were identified as previously described ${ }^{38}$ and performed by MultiS Fast Mutagenesis kit V2 
(Vazyme, Nanjing, China). The SH2, EEP and P rich domains of SHIP1 were generated from a full-length SHIP1 plasmid. The SHIP1- $\triangle$ SH2, SHIP1- $\triangle$ EEP and SHIP1- $\triangle \mathrm{P}$ rich plasmid were generated by deleting key domains as described above. All clones were sequenced and the expression was confirmed by immunoblotting transiently transfected 293T (ATCC CRL-11268) cell lysates with anti-Tag antibodies. RAW264.7 cells were transiently transfected with plasmids or blank vector by using Lipofectamine 2000 (Invitrogen) as a previously described ${ }^{66}$. All the siRNAs used were purchased from RiboBio (Guangzhou, China). Negative control siRNA sequence (siN05815122147) was supplied by RiboBio. The target sequences of siRNAs are listed as Supplementary Table 2.

\section{Retrovirus-mediated gene transduction}

For making RAW264.7 stable cell lines, the cDNA encoding full-length mouse SLAMF7 (NCBI Gene ID: 75345) was cloned into pSin-EF2-puro-oligo transfer plasmid. For deletion of SLAMF7 gene in RAW264.7, sgRNAs were cloned into pXPR_001 plasmid according to Zhang's lab lentiCRISPR system ${ }^{67}$. Retrovirus expressing the appropriate alleles was produced by 293FT cells co-transfected with the packaging system composed of psPAX2 packaging plasmid and pMD2.G envelope plasmid. $48 \mathrm{~h}$ after transfection, viral supernatants were collected and filtered and $2 \mathrm{ml}$ of viral supernatant was used to transducer $5 \times 10^{6}$ RAW264.7 cells in the presence of $8 \mu \mathrm{g} / \mathrm{ml}$ of polybrene (Sigma, St. Louis, MO). $24 \mathrm{~h}$ post viral infection, RAW264.7 cells were selected by puromycin (InvivoGen, CA, USA) for 3 days and then were used for the selection of a single clone. Cells against puromycin were subjected for single clone by folds dilution. The positive percentage of SLAMF7 were detected by flow cytometry. The knockout of SLAMF7 was confirmed by immunoblot compared to wild-type RAW264.7 cells.

\section{Confocal microscopy and histological staining}

Cells were grown on Glass Bottom Cell Culture Dish were fixed with $4 \%$ paraformaldehyde (BBI Life Science, Shanghai, China) followed by membrane permeabilization, blocking and antibody incubation. Nuclei were stained with 4,6-diamidino-2-phenylindole (DAPI, Invitrogen) and mounted with ProLong Gold antifade reagent from Invitrogen and viewed by confocal microscopy (Zeiss, LSM780, German). For histological section staining, mouse lung tissue samples were fixed in 4\% PFA for $24 \mathrm{~h}$ and embedded in paraffin. Four- $\mu$ m-thick sections were deparaffinized and stained with hematoxylin and eosin to determine organ damage and leukocyte infiltration.

\section{BMDM and human monocytes isolation}

Murine BMDMs were obtained as previously described ${ }^{11}$. Briefly cells were maintained in DMEM supplemented with $10 \%$ FBS and 10\% L929 cell supernatant as conditioned medium providing macrophage colony stimulating factor. BMDMs were obtained as a homogeneous population of adherent cells after 7 days culture. The purity of the differentiation method was $>95 \%$ as a routinely confirmed by flow cytometry. Human monocytes were isolated from peripheral venous blood from healthy subjects. Monocytes were isolated from the peripheral blood mononuclear cell (PBMC) layer according to Ficoll manufacturers (TBD, Tianjin, China). An ammonium chloride-based lysis (BD Biosciences) was 
performed to remove erythrocytes. PBMCs were then stained in PBS containing 1\% BSA with monoclonal antibodies to CD14 (BD Biosciences) for sorting. Purified monocytes were cultured at $1 \times 10^{6} \mathrm{cells} / \mathrm{ml}$ overnight before use.

\section{Immunoblot analysis and immunoprecipitation assay}

Cells samples were lysed in RIPA buffer with protease and phosphatase inhibitor cocktail (Sigma), and then the protein extracts were separated by SDS-PAGE electrophoresis and transferred to a polyvinylidene fluoride or nitrocellulose membranes. The membranes were incubated overnight at $4{ }^{\circ} \mathrm{C}$ with antibodies against SLAMF7 (MAB4628), SLAMF7 (SC-46517, Santa Cruz), SHIP1 (D1163, \#2728), TRAF6 (sc-7221, Santa Cruz), AKT (\#9272), Ubiquitination (P4D1,\#3936T), HA-Tag (C29F4, \#3724S), Flag-Tag (DYKDDDK, \#2368S), Myc-Tag (\#2276), $\beta$-actin or the phosphorylation of IKKX/ $\beta$ (16A6, \#2697S), SHIP1 (\#3941S), AKT (\#2965S), JNK (\#4668S), ERK (\#4370S), p-38 (\#9215S). Then the membranes were incubated with appropriate HRP-conjugated secondary antibodies (R\&D) followed by the visualization with PlusECL (KeyGEN BioTECH). Alternatively, membranes were detected with IRDye 800CW conjugated anti-rabbit IgG or IRDye $680 \mathrm{CW}$ conjugated anti-mouse IgG secondary antibodies (LI-COR Biosciences, Lincoln, NE), and visualized using Odyssey infrared imaging system (LI-COR Biosciences). All antibodies were purchased from Cell Signaling Technology (Beverly, MA, USA) or Abcam if otherwise indicated. For endogenous immunoprecipitation, the protein $\mathrm{G}$ plus protein $\mathrm{A}$ agarose suspension was purchased from Calbiochem (USA). The anti-Flag M2 affinity gel (\#A2220, Sigma) and the anti-HA agarose beads (AT0079, CMCTAG) were used for the other immunoprecipitation assay.

\section{Reverse transcription and quantitative PCR assay}

RNA was extracted from whole cell lysates with TRIzol (Invitrogen) and was reversely transcribed to cDNA with a First Strand cDNA Synthesis Kit (Thermo Fisher Scientific, Waltham, MA). Quantitative real-time PCR was performed in triplicate determinants with a CFX96 RT-PCR Detection System (Bio-Rad). Primary transcripts were measured with primers that amplify either exon-intron junctions or intronic sequences. Threshold cycle numbers were normalized to triplicate samples amplified with primers specific for $\beta$ actin. The primer sequences used in this study are listed in the Supplementary Table 3.

\section{Statistical analysis}

Statistical analysis was performed using Prism version 7 (Graphpad Software, San Diego, CA), and data are shown as mean \pm s.e.m; unless otherwise stated. For two-group comparisons, a two-tailed unpaired Student's $t$ test was used. Two-way non-parametric ANOVA was used by Tukey's multiple comparisons multiple comparison test for multi-group comparisons. All FACS analysis, immunofluorescence analysis and western blots were repeated at least in three independent experiments.

\section{Declarations}

Acknowledgements 
This work was supported by grants from National Natural Science Foundation of China (82072062), National Science and Technology Key Projects for Major Infectious Diseases (2017ZX10302301-002), Guangzhou Science and Technology Planning Project (201704020226 and 201604020006), National Key Research and Development Program of China (2016YFC1200105), The Three Major Scientific Research Projects of Sun Yat-sen University (20200326236), Guangdong Scientific and Technological Research Project for COVID-19 containment (2020A111128022, 2020B111112003), Guangdong Scientific and Technological Research for COVID-19 (202020012612200001), Zhuhai Scientific and Technological Research Project for COVID-19 containment (ZH22036302200029PWC).

\section{Author contributions}

Y.W., J.L., M.L., S.M. and X.H. performed the experiments; M.W., L.L., S.G., H.Z. and X.H. provided scientific expertise; Y.W., J.L., M.L. and X.H. designed the study, interpreted the data and wrote the manuscript; All authors read the final version of the manuscript and approved the submission.

\section{References}

1. Angus, D.C. \& Van Der Poll, T. Severe sepsis and septic shock. N. Engl. J. Med. 369, 840-851 (2013).

2. Vincent, J.-L., Opal, S.M., Marshall, J.C. \& Tracey, K.J. Sepsis definitions: time for change. Lancet 381, 774 (2013).

3. Marshall, J.C. Sepsis-3: what is the meaning of a definition? Crit. Care Med. 44, 1459-1460 (2016).

4. Cecconi, M., Evans, L., Levy, M. \& Rhodes, A. Sepsis and septic shock. Lancet 392, 75-87 (2018).

5. Broz, P. \& Monack, D.M. Newly described pattern recognition receptors team up against intracellular pathogens. Nat. Rev. Immunol. 13, 551-565 (2013).

6. Williams, D.L. et al. Modulation of tissue Toll-like receptor 2 and 4 during the early phases of polymicrobial sepsis correlates with mortality. Crit Care Med 31, 1808-1818 (2003).

7. Tidswell, M. et al. Phase 2 trial of eritoran tetrasodium (E5564), a toll-like receptor 4 antagonist, in patients with severe sepsis. Crit Care Med 38, 72-83 (2010).

8. Zhang, Y. et al. Activation of vascular endothelial growth factor receptor-3 in macrophages restrains TLR4-NF-kappaB signaling and protects against endotoxin shock. Immunity 40, 501-514 (2014).

9. Xu, S. et al. Constitutive MHC class I molecules negatively regulate TLR-triggered inflammatory responses via the Fps-SHP-2 pathway. Nat Immunol 13, 551-559 (2012).

10. Lee, J.K., Mathew, S.O., Vaidya, S.V., Kumaresan, P.R. \& Mathew, P.A. CS1 (CRACC, CD319) induces proliferation and autocrine cytokine expression on human B lymphocytes. $J$ Immuno/ 179, 46724678 (2007).

11. Zhu, S. et al. Signaling Lymphocytic Activation Molecule Family-7 Alleviates Corneal Inflammation by Promoting M2 Polarization. J Infect Dis (2020).

12. De Calisto, J. et al. SAP-Dependent and -Independent Regulation of Innate T Cell Development Involving SLAMF Receptors. Front Immuno/ 5, 186 (2014). 
13. Kim, J.R., Horton, N.C., Mathew, S.O. \& Mathew, P.A. CS1 (SLAMF7) inhibits production of proinflammatory cytokines by activated monocytes. Inflamm Res 62, 765-772 (2013).

14. Stark, S. \& Watzl, C. 2B4 (CD244), NTB-A and CRACC (CS1) stimulate cytotoxicity but no proliferation in human NK cells. Int. Immunol. 18, 241-247 (2006).

15. Cruz-Munoz, M.E., Dong, Z., Shi, X., Zhang, S. \& Veillette, A. Influence of CRACC, a SLAM family receptor coupled to the adaptor EAT-2, on natural killer cell function. Nat. Immunol. 10, 297-305 (2009).

16. Chen, J. et al. SLAMF7 is critical for phagocytosis of haematopoietic tumour cells via Mac-1 integrin. Nature 544, 493-497 (2017).

17. Malaer, J.D. \& Mathew, P.A. CS1 (SLAMF7, CD319) is an effective immunotherapeutic target for multiple myeloma. Am J Cancer Res 7, 1637-1641 (2017).

18. Lamb, Y.N. Elotuzumab: A Review in Relapsed and/or Refractory Multiple Myeloma. Drugs 78, 14811488 (2018).

19. Chim, C.S. et al. Management of relapsed and refractory multiple myeloma: novel agents, antibodies, immunotherapies and beyond. Leukemia 32, 252-262 (2018).

20. O'Connell, P. et al. SLAMF7 Is a Critical Negative Regulator of IFN-alpha-Mediated CXCL10 Production in Chronic HIV Infection. J Immuno/ 202, 228-238 (2019).

21. Pierrakos, C. \& Vincent, J.L. Sepsis biomarkers: a review. Crit. Care 14, R15 (2010).

22. Harrison, C. Sepsis: calming the cytokine storm. Nat. Rev. Drug Discov. 9, 360-361 (2010).

23. Biswas, S.K. \& Lopez-Collazo, E. Endotoxin tolerance: new mechanisms, molecules and clinical significance. Trends Immunol. 30, 475-487 (2009).

24. Meisner, M., Tschaikowsky, K., Palmaers, T. \& Schmidt, J. Comparison of procalcitonin (PCT) and Creactive protein (CRP) plasma concentrations at different SOFA scores during the course of sepsis and MODS. Crit. Care 3, 45 (1999).

25. Kawai, T. \& Akira, S. Toll-like Receptors and Their Crosstalk with Other Innate Receptors in Infection and Immunity. Immunity 34, 637-650 (2011).

26. Fitzgerald, K.A. et al. Mal (MyD88-adapter-like) is required for Toll-like receptor-4 signal transduction. Nature 413, 78-83 (2001).

27. Nowill, A.E. et al. Immune Response Resetting in Ongoing Sepsis. J Immunol 203, 1298-1312 (2019).

28. Strassheim, D. et al. Phosphoinositide 3-Kinase and Akt Occupy Central Roles in Inflammatory Responses of Toll-Like Receptor 2-Stimulated Neutrophils. J. Immuno/ 172, 5727-5733 (2004).

29. Engel, P., Eck, M.J. \& Terhorst, C. The SAP and SLAM families in immune responses and X-linked lymphoproliferative disease. Nat. Rev. Immunol. 3, 813-821 (2003).

30. Guo, H., Cruz-Munoz, M.E., Wu, N., Robbins, M. \& Veillette, A. Immune cell inhibition by SLAMF7 is mediated by a mechanism requiring src kinases, CD45, and SHIP-1 that is defective in multiple myeloma cells. Mol. Cell. Biol. 35, 41-51 (2015). 
31. Tassi, I. \& Colonna, M. The cytotoxicity receptor CRACC (CS-1) recruits EAT-2 and activates the PI3K and phospholipase Cgamma signaling pathways in human NK cells. J Immuno/ 175, 7996-8002 (2005).

32. Lee, S.Y., Lee, S.Y. \& Choi, Y. TRAF-interacting protein (TRIP): a novel component of the tumor necrosis factor receptor (TNFR)- and CD30-TRAF signaling complexes that inhibits TRAF2-mediated NF-kappaB activation. J Exp Med 185, 1275-1285 (1997).

33. Dong, W. et al. The IRAK-1-BCL10-MALT1-TRAF6-TAK1 cascade mediates signaling to NF-kappaB from Toll-like receptor 4. J Biol Chem 281, 26029-26040 (2006).

34. Li, M. et al. T-cell immunoglobulin and ITIM domain (TIGIT) receptor/poliovirus receptor (PVR) ligand engagement suppresses interferon-ү production of natural killer cells via $\beta$-arrestin 2-mediated negative signaling. J. Biol. Chem. 289, 17647-17657 (2014).

35. Deng, L. et al. Activation of the IKB Kinase Complex by TRAF6 Requires a Dimeric UbiquitinConjugating Enzyme Complex and a Unique Polyubiquitin Chain. Cell 103, 351-361 (2000).

36. Yang, W.L. et al. The E3 ligase TRAF6 regulates Akt ubiquitination and activation. Science 325, 11341138 (2009).

37. Boles, K.S., Stepp, S.E., Bennett, M., Kumar, V. \& Mathew, P.A. 2B4 (CD244) and CS1: novel members of the CD2 subset of the immunoglobulin superfamily molecules expressed on natural killer cells and other leukocytes. Immunol Rev 181, 234-249 (2001).

38. Chen, J. et al. SLAMF7 is critical for phagocytosis of haematopoietic tumour cells via Mac-1 integrin. Nature 544, 493-497 (2017).

39. Bao, M. et al. CD2AP/SHIP1 complex positively regulates plasmacytoid dendritic cell receptor signaling by inhibiting the E3 ubiquitin ligase Cbl. J. Immunol. 189, 786-792 (2012).

40. Fernandes, S., lyer, S. \& Kerr, W.G. Role of SHIP1 in cancer and mucosal inflammation. Ann NY Acad Sci 1280, 6-10 (2013).

41. Dejager, L., Pinheiro, I., Dejonckheere, E. \& Libert, C. Cecal ligation and puncture: the gold standard model for polymicrobial sepsis? Trends Microbio/ 19, 198-208 (2011).

42. Kumaresan, P.R., Lai, W.C., Chuang, S.S., Bennett, M. \& Mathew, P.A. CS1, a novel member of the CD2 family, is homophilic and regulates NK cell function. Mol. Immunol. 39, 1-8 (2002).

43. Campbell, K.S., Cohen, A.D. \& Pazina, T. Mechanisms of NK Cell Activation and Clinical Activity of the Therapeutic SLAMF7 Antibody, Elotuzumab in Multiple Myeloma. Front Immuno/ 9, 2551 (2018).

44. Raedler, L.A. Empliciti (Elotuzumab): First SLAMF7 Antibody Therapy Approved for the Treatment of Patients with Previously Treated Multiple Myeloma. Am Health Drug Benefits 9, 74-77 (2016).

45. Lonial, S. et al. Update on elotuzumab, a novel anti-SLAMF7 monoclonal antibody for the treatment of multiple myeloma. Expert Opin. Biol. Ther. 16, 1291-1301 (2016).

46. van de Donk, N.W. et al. Clinical efficacy and management of monoclonal antibodies targeting CD38 and SLAMF7 in multiple myeloma. Blood 127, 681-695 (2016). 
47. Woo, J. et al. PDL241, a novel humanized monoclonal antibody, reveals CD319 as a therapeutic target for rheumatoid arthritis. Arthritis Res. Ther. 15, R207 (2013).

48. Burbage, M. et al. Tuning of in vivo cognate B-T cell interactions by Intersectin 2 is required for effective anti-viral B cell immunity. Elife 7 (2018).

49. van der Heide, V., Mohnle, P., Rink, J., Briegel, J. \& Kreth, S. Down-regulation of MicroRNA-31 in CD4+ T Cells Contributes to Immunosuppression in Human Sepsis by Promoting TH2 Skewing. Anesthesiology 124, 908-922 (2016).

50. Amagai, Y. et al. Oncolytic Activity of a Recombinant Measles Virus, Blind to Signaling Lymphocyte Activation Molecule, Against Colorectal Cancer Cells. Sci. Rep. 6, 24572 (2016).

51. Berger, S.B. et al. SLAM is a microbial sensor that regulates bacterial phagosome functions in macrophages. Nat. Immunol. 11, 920-927 (2010).

52. Shin, J.-S., Gao, Z. \& Abraham, S.N. Involvement of cellular caveolae in bacterial entry into mast cells. Science 289, 785-788 (2000).

53. Hashiguchi, T. et al. Structure of the measles virus hemagglutinin bound to its cellular receptor SLAM. Nat. Struct. Mol. Biol. 18, 135-141 (2011).

54. Kumaresan, P.R., Lai, W.C., Chuang, S.S., Bennett, M. \& Mathew, P.A. CS1, a novel member of the CD2 family, is homophilic and regulates NK cell function. Mol Immunol 39, 1-8 (2002).

55. Ozes, O.N. et al. NF-kB activation by tumour necrosis factor requires the Akt serine-threonine kinase. Nature 401, 82-85 (1999).

56. Romashkova, J.A. \& Makarov, S.S. NF-KB is a target of AKT in anti-apoptotic PDGF signalling. Nature 401, 86-90 (1999).

57. Rommel, C. et al. Differentiation stage-specific inhibition of the Raf-MEK-ERK pathway by Akt. Science 286, 1738-1741 (1999).

58. Zimmermann, S. \& Moelling, K. Phosphorylation and regulation of Raf by Akt (protein kinase B). Science 286, 1741-1744 (1999).

59. Sly, L.M., Rauh, M.J., Kalesnikoff, J., Song, C.H. \& Krystal, G. LPS-induced upregulation of SHIP is essential for endotoxin tolerance. Immunity 21, 227-239 (2004).

60. An, H. et al. Src homology 2 domain-containing inositol-5-phosphatase 1 (SHIP1) negatively regulates TLR4-mediated LPS response primarily through a phosphatase activity- and PI-3Kindependent mechanism. Blood 105, 4685-4692 (2005).

61. Veillette, A. Immune regulation by SLAM family receptors and SAP-related adaptors. Nat. Rev. Immunol. 6, 56-66 (2006).

62. Boone, D.L. et al. The ubiquitin-modifying enzyme A20 is required for termination of Toll-like receptor responses. Nat. Immunol. 5, 1052 (2004).

63. Trompouki, E. et al. CYLD is a deubiquitinating enzyme that negatively regulates NF-KB activation by TNFR family members. Nature 424, 793-796 (2003). 
64. Chen, H. et al. Hsp70 inhibits lipopolysaccharide-induced NF-KB activation by interacting with TRAF6 and inhibiting its ubiquitination. FEBS Lett. 580, 3145-3152 (2006).

65. Kim, E. et al. Inositol polyphosphate multikinase promotes Toll-like receptor-induced inflammation by stabilizing TRAF6. Sci Adv 3, e1602296 (2017).

66. Yang, K. et al. miR-155 suppresses bacterial clearance in Pseudomonas aeruginosa-induced keratitis by targeting Rheb. J. Infect. Dis. 210, 89-98 (2014).

67. Ran, F.A. et al. Genome engineering using the CRISPR-Cas9 system. Nat. Protoc. 8, 2281-2308 (2013).

\section{Figures}


a

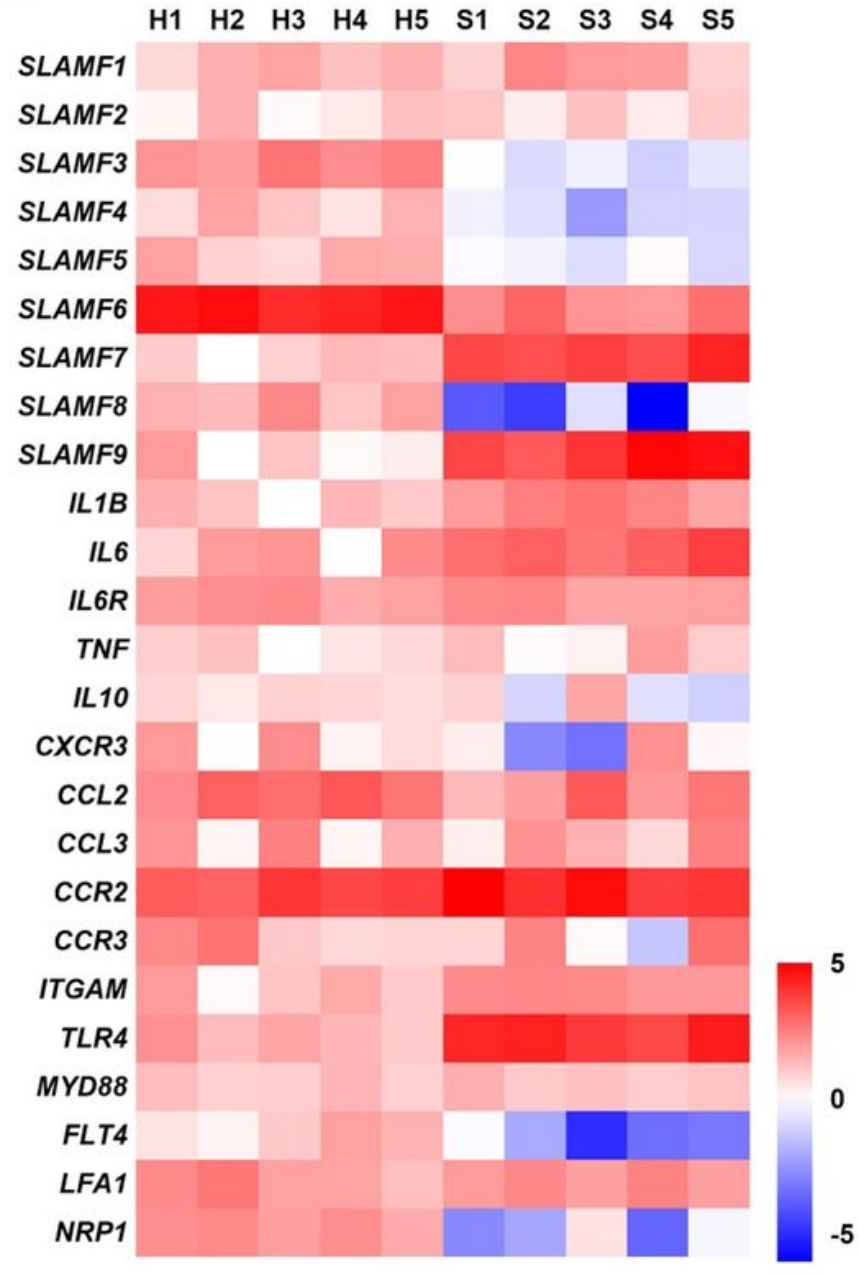

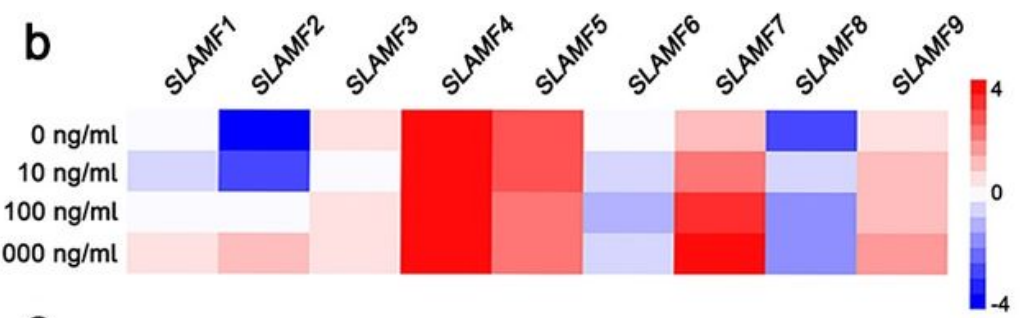

C
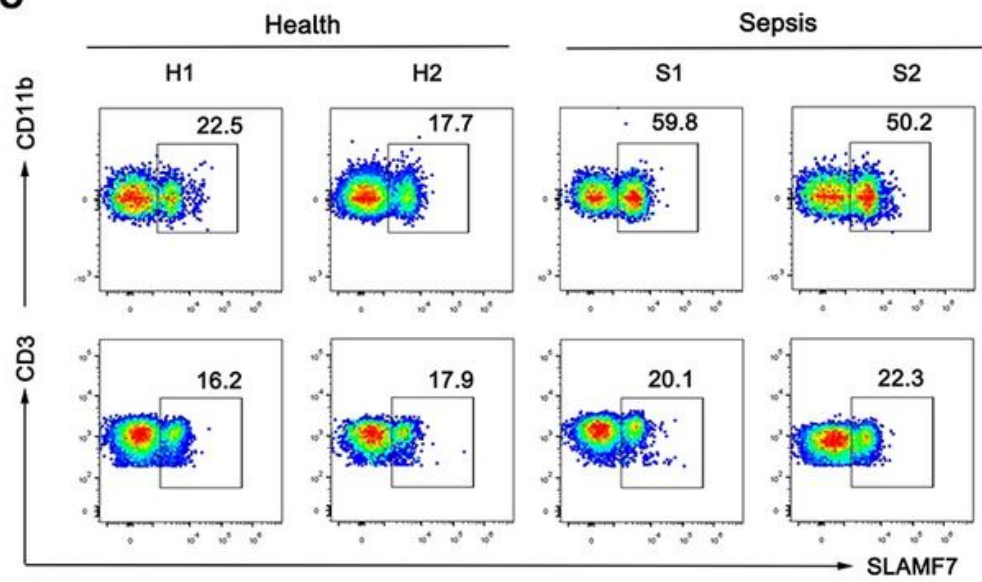

d

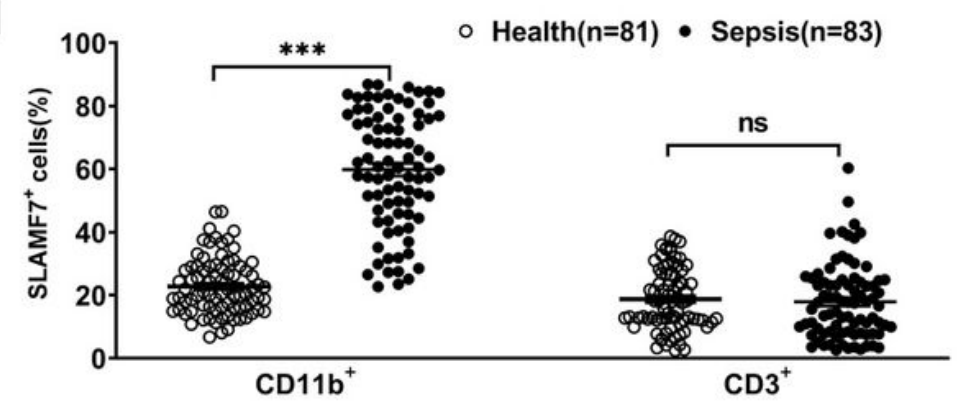

e

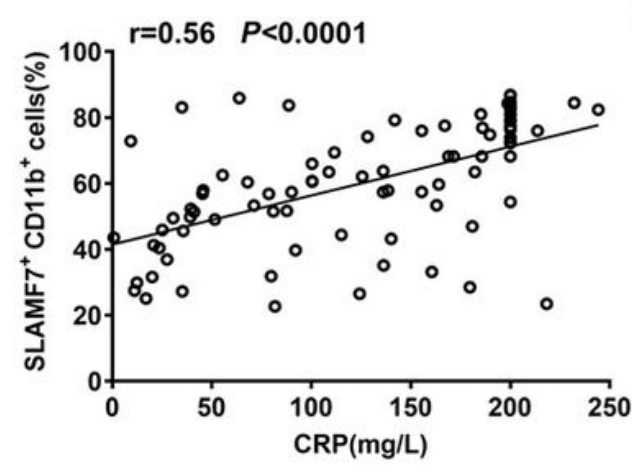

f

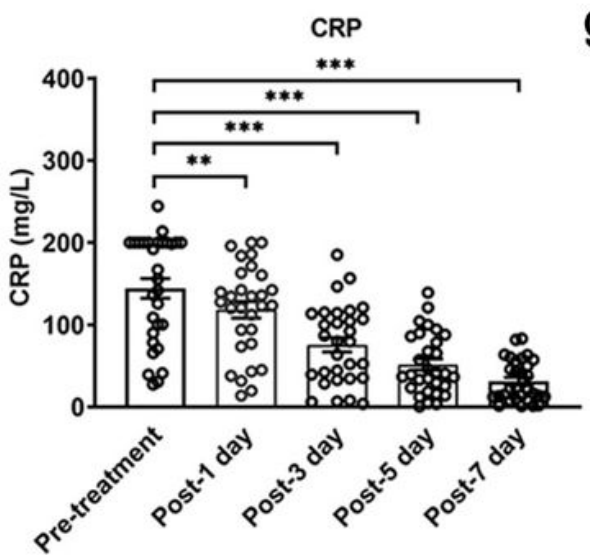

g

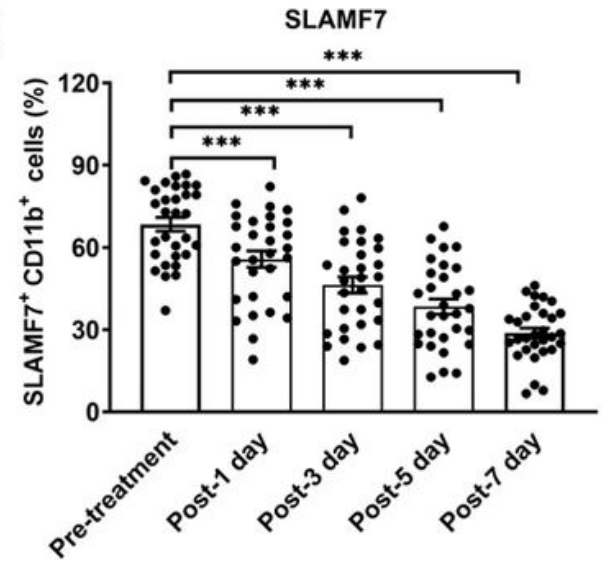

Figure 1

SLAMF7 expression is associated with sepsis. a, mRNA expression levels of inflammation-related molecules in peripheral blood mononuclear cells (PBMC) of sepsis patients (S1-S5) were compared with that in healthy donors $(\mathrm{H} 1-\mathrm{H} 5)$. The fold change for each gene was normalized to $\beta$-actin expression. $b$, Expression of SLAM family members in human monocyte-derived macrophages after LPS stimulation for $12 \mathrm{~h}$ with different doses. $\mathrm{c}, \mathrm{d}$, The percentage of SLAMF7+ cells in CD11b+ or CD3+ cells subset from human healthy donors $(n=81)$ or patients with sepsis $(n=83)$. e, The correlation of C-reactive protein (CRP) 
and SLAMF7 expression in sepsis patients. $\mathrm{f}$, Protein levels of CRP in the serum of sepsis patients were analyzed by ELISA before clinical treatment (pre-treatment) or at 1, 3, 5 and 7 days post-treatment. $g$,

Percentage of SLAMF7+ cells in CD11b+ subsets was analyzed by flow cytometry before treatment and at 1, 3, 5 and 7 days post-treatment. Data are the mean \pm SEM and represent three individual experiments, ns, not significant; **, $\mathrm{P}<0.01$; ***, $\mathrm{P}<0.001$.

a

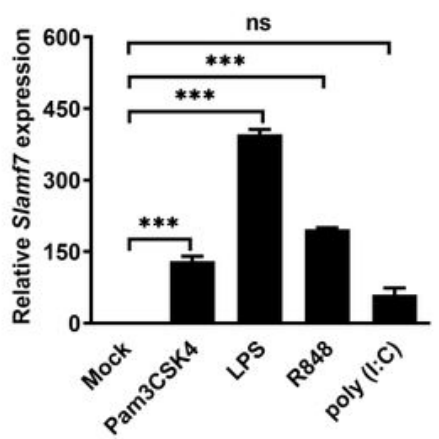

d

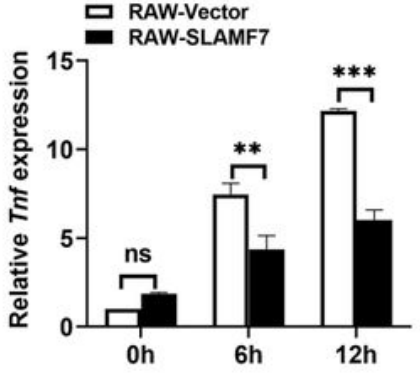

e
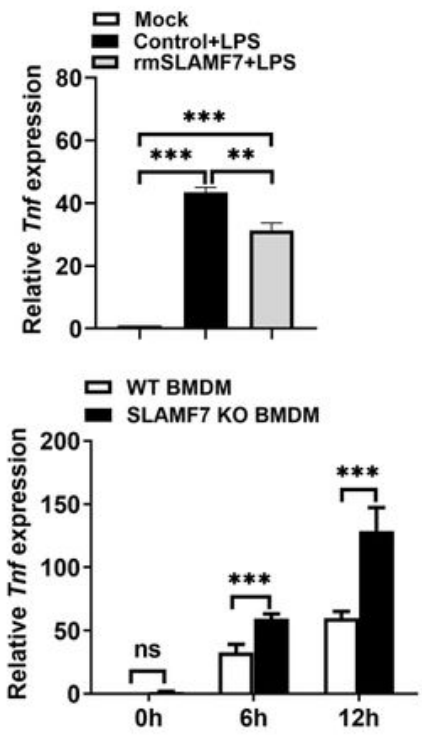

g

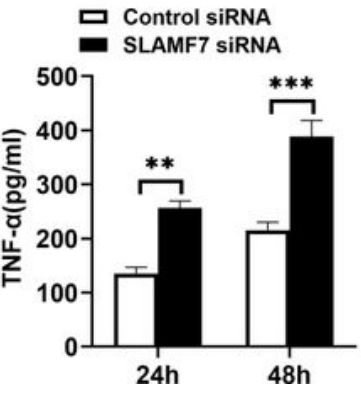

b
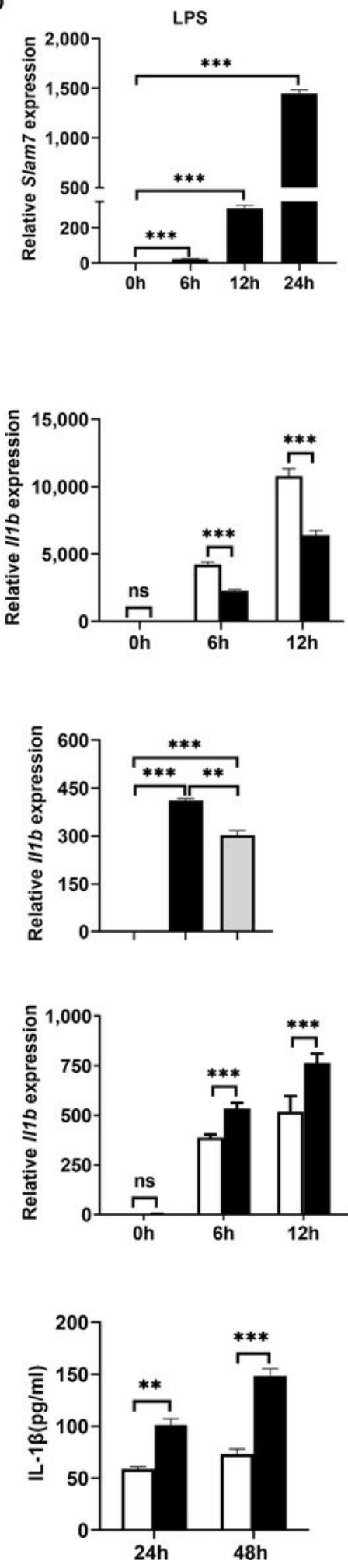

C

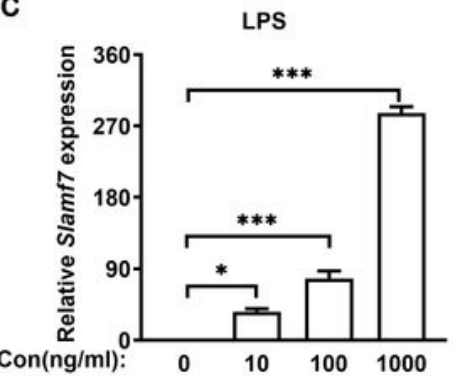

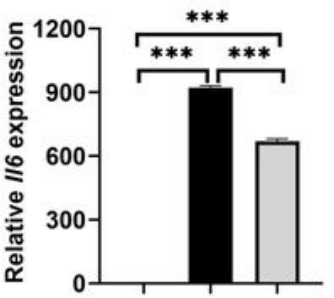
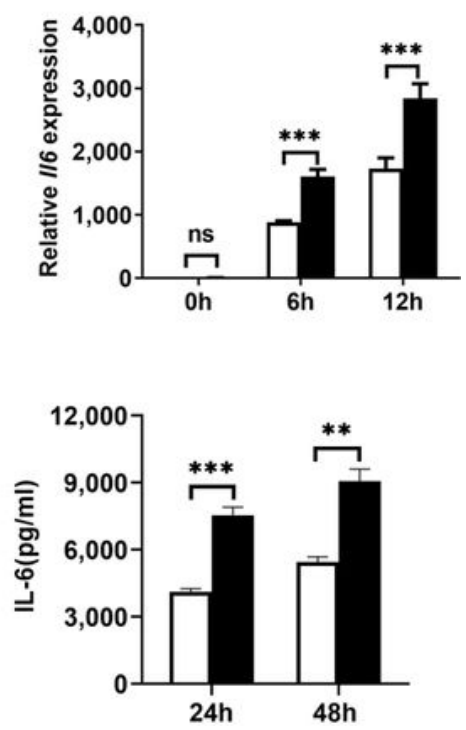

Figure 2 
SLAMF7 negatively regulates the production of pro-inflammatory cytokines. a, mRNA levels of Slamf7 in RAW264.7 cells were examined by real-time PCR after stimulation with Pam3Csk4 (TLR1/2 ligand), LPS (TLR4 ligand), R848 (TLR7/8 ligand) and poly (I:C) (TLR3 ligand) for 24 h. b, c, mRNA levels of Slamf7 were examined in RAW264.7 cells after LPS treatment at indicated time (b) or concentrations (c). d, RAW264.7 cells stably expressing SLAMF7 (RAW-SLAMF7) and control RAW-Vector (RAW-Vector) cells were constructed. mRNA levels of Tnf, $\| 1 \mathrm{~b}$ and $\| 6$ in RAW-SLAMF7 cells vs RAW-Vector cells were analyzed at $0 \mathrm{~h}, 6 \mathrm{~h}$ and $12 \mathrm{~h}$ after LPS stimulation. e, Gene expression of Tnf, $\| 1 \mathrm{~b}$, and $\| 6 \mathrm{in}$ BMDM pretreatment with rmSLAMF7 $(1 \mathrm{ug} / \mathrm{ml})$ vs control $(0.9 \% \mathrm{NaCl})$ at $6 \mathrm{~h}$ after LPS stimulation. $\mathrm{f}$, Tnf, $\| 1 \mathrm{~b}$ and $\| 6$ gene expression of WT and SLAMF7 KO BMDM after LPS stimulation at $0 \mathrm{~h}, 6 \mathrm{~h}$ and $12 \mathrm{~h} . \mathrm{g}$, The protein level of IL-1 $\beta$ and IL-6 in the supernatant of BMDM transfected with SLAMF7 siRNA or Control siRNA, followed by LPS stimulation for $24 \mathrm{~h}$ and $48 \mathrm{~h}$. Data are the mean \pm SEM and represent three individual experiments. ns, not significant; $*, P<0.05$; $* *, P<0.01$; ***, $\mathrm{P}<0.001$.

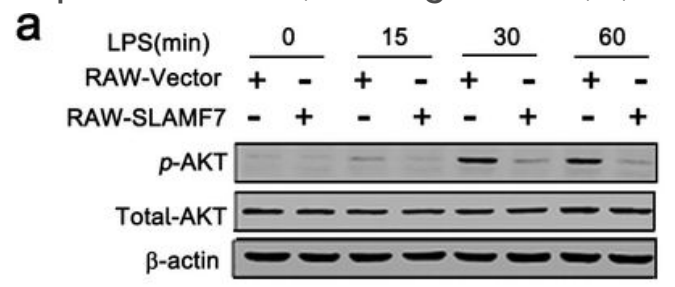

d

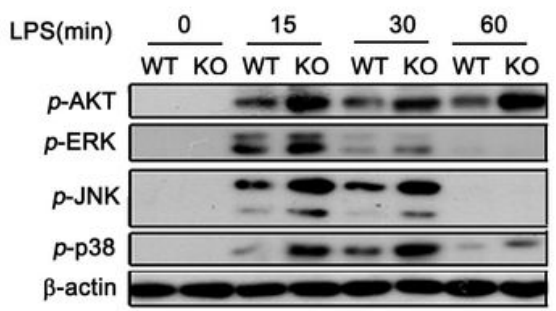

g

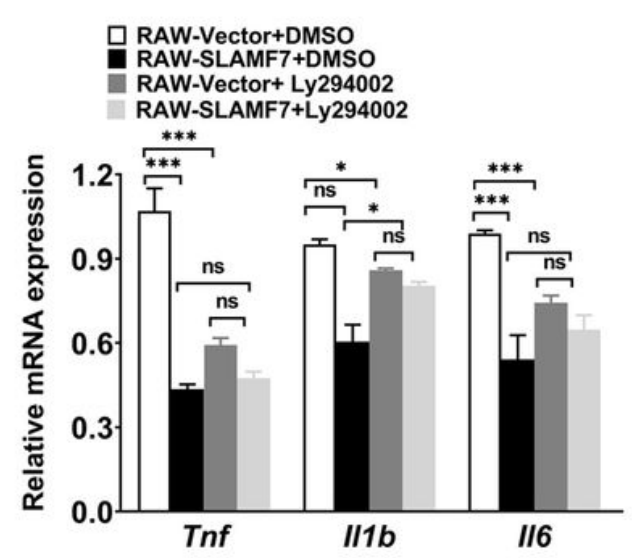

j

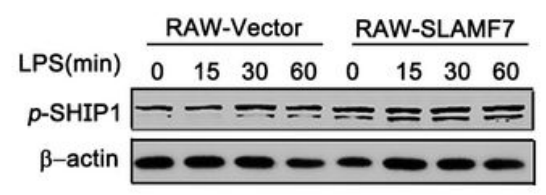

b

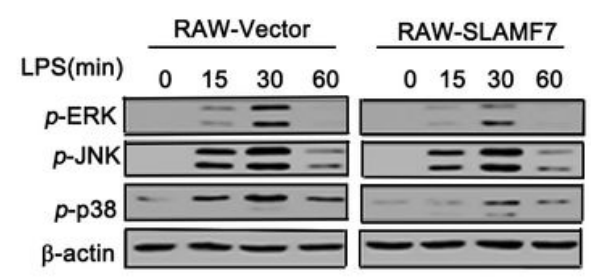

e

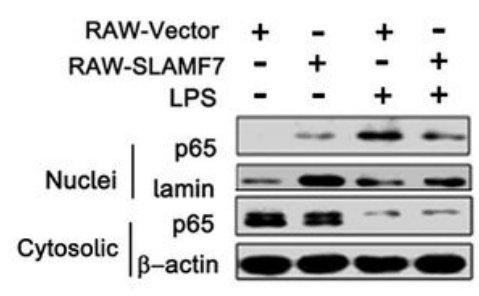

h
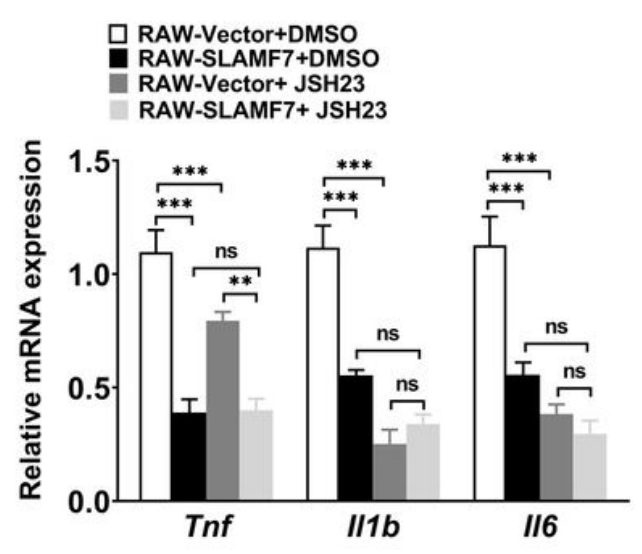

C
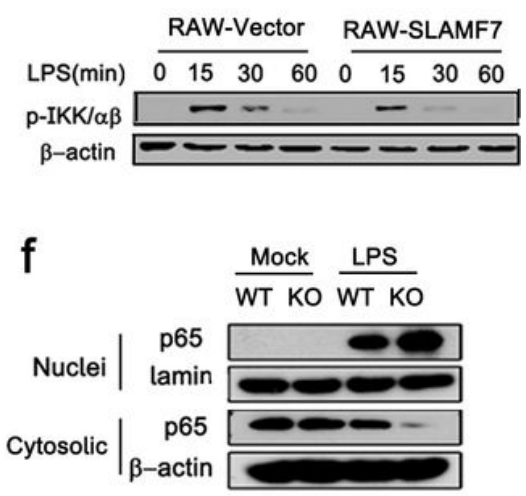

i

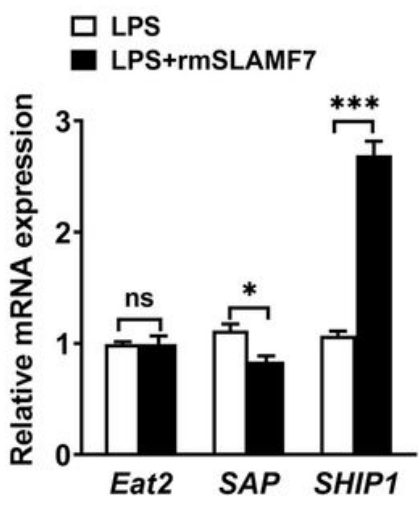

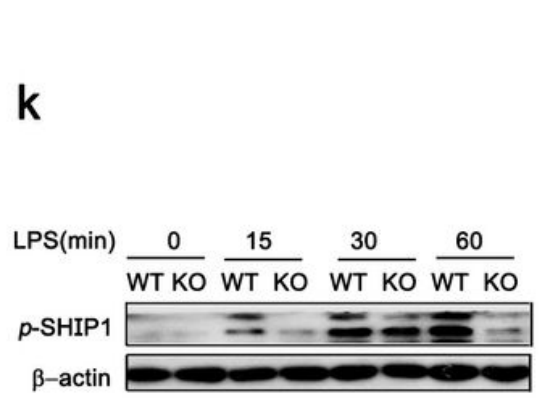

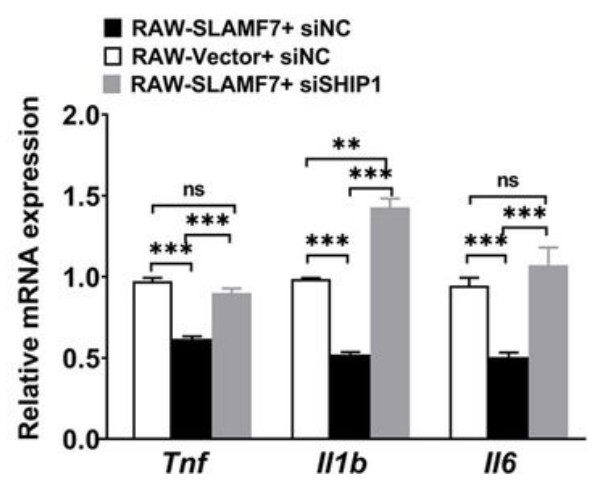




\section{Figure 3}

SLAMF7 attenuates MAPKs/NF-kB signaling pathways by activating SHIP1. a-c, Phosphorylation of AKT (a), MAPKs (b) and IKKa/ $\beta$ (c) in RAW-SLAMF7 vs RAW-Vector cells were examined by Western blot after LPS stimulation at indicated time points. $d$, WT vs SLAMF7 KO BMDM were challenged with LPS for indicated time points, then phosphorylated MAPKs and AKT were determined by Western blot. e, $f$, The protein level of NF-KB p65 subunit in nuclei and cytosolic fraction of LPS-treated RAW-Vector vs RAWSLAMF7 cells (e) or WT vs SLAMF7 KO BMDM (f). $g$, h, The expression of Tnf, $\| 1 \mathrm{~b}$ and $\| 6$ of RAW264.7 cells pre-treatment with an inhibitor targeting PI3K/Akt (Ly294002) (g) and NF-KB (JSH23) (h), followed by LPS stimulation for $6 \mathrm{~h}$. i, The expression level of Eat2, Sap and Ship1 of BMDM after stimulated with rmSLAMF7, followed by LPS stimulation. j, k, Immunoblot analysis of phosphorylated SHIP1 in RAWVector vs RAW-SLAMF7 cells (j) or WT vs SLAMF7 KO BMDM ( $k$ ) stimulated with LPS for 0min,15min,30min,60 min. I, The expression of Tnf, II1 b and II6 of RAW264.7 cells after pre-transfected with SHIP1 siRNA, followed by LPS stimulation for $6 \mathrm{~h}$. Data are the mean \pm SEM and represent three

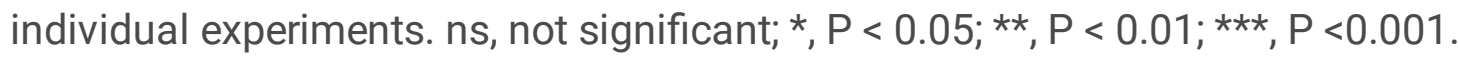


a

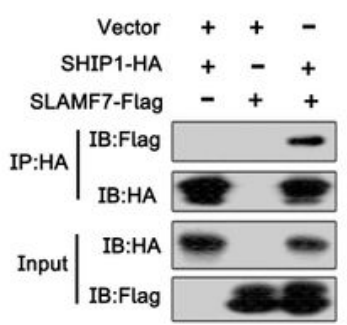

d

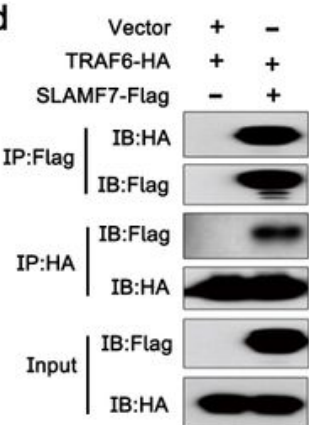

g

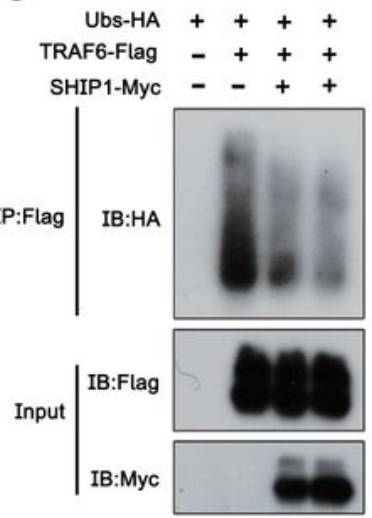

b

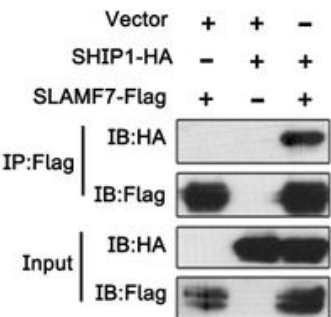

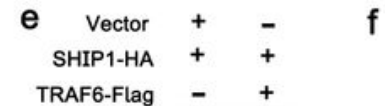

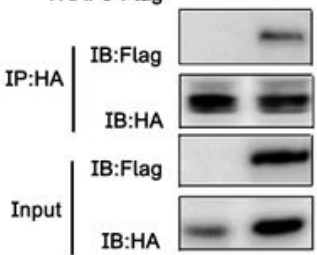

C
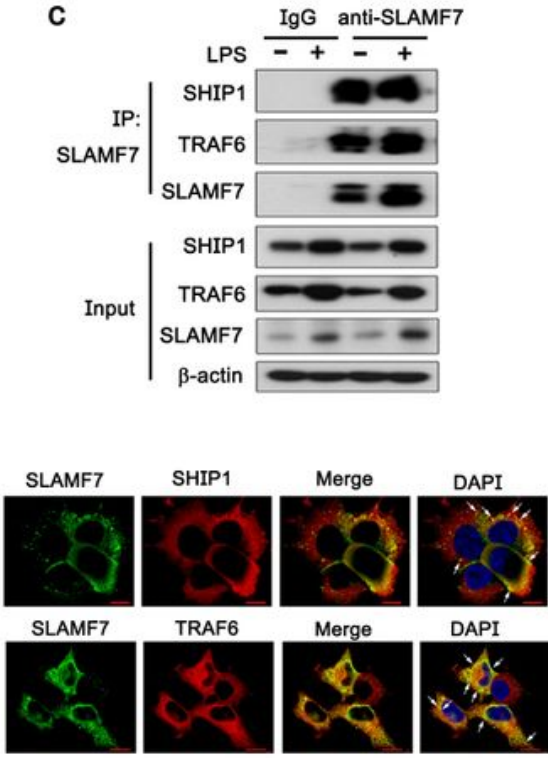

Ubs-HA $\frac{\text { SHIP1+SLAMF7 }}{++---}$

K48 Ubs-HA - + - -

K63 Ubs-HA - - + -

K48R Ubs-HA - - - +

K63R Ubs-HA - - - +

TRAF6-Flag +++++
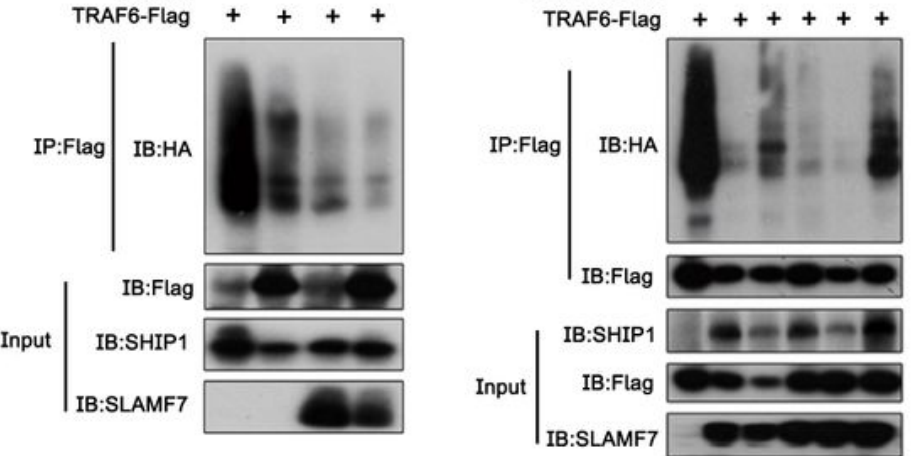

Control siRnA +--+-

SLAMF7 siRNA-1 - + - + -

SLAMF7 SiRNA-2 - ++-+

MG132 + + + - -

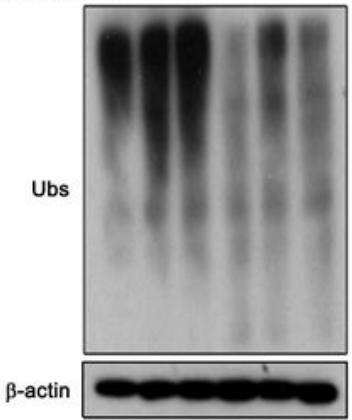

k

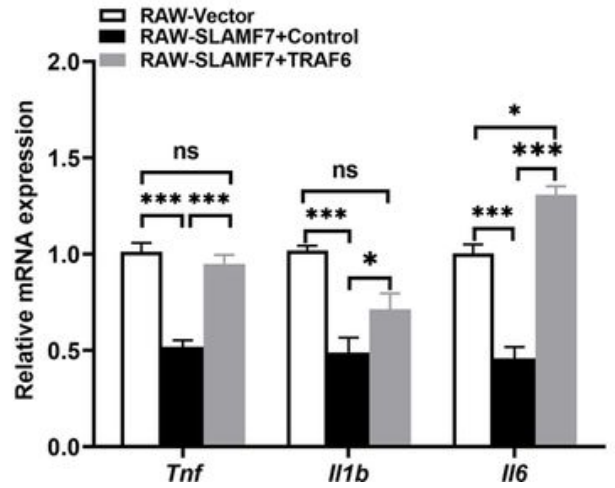

Figure 4

SLAMF7 co-operates with SHIP1 to inhibit TRAF6 K63 ubiquitination. a, b, Immunoprecipitation using anti-Flag or anti-HA antibodies from lysate of 293T cells transfected with Flag-tagged SLAMF7 alone, Flag-tagged SLAMF7 and HA-tagged SHIP1, c, Immunoassay of lysate of RAW264.7 cells stimulated with LPS, followed by immunoprecipitation (IP) with IgG or anti-SLAMF7 and immunoblot analysis (IB) with anti-TRAF6 or anti-SHIP1. d, e, Immunoprecipitation using anti-Flag or anti-HA antibodies from lysate of 
293T cells transfected with HA-tagged TRAF6 and Flag-tagged SLAMF7 (d) or HA-tagged SHIP1 and Flag-tagged TRAF6 (e). f, Confocal microscopy of 293T cells co-transfected with Flag-tagged SLAMF7 and HA-tagged SHIP1 (above lane) or HA-tagged TRAF6 (below lane). White arrows represent the colocalization. Scale bars, $10 \mu \mathrm{m}$. $\mathrm{g}$, Immunoblot of TRAF6-Ubs of precipitation with anti-HA antibodies from lysate of 293T cells transfected with Flag-tagged TRAF6, HA-tagged Ubs, Myc-tagged SHIP1. h, Immunoblot analysis of TRAF6 ubiquitination from precipitation of lysate of 293T cells transfected with Flag-tagged TRAF6, HA-tagged ubiquitin with or without SLAMF7 and SHIP1. i, Immunoblot analysis of lysate of ubiquitination of TRAF6 in 293T cells transfected with HA-tagged ubiquitin, HA-tagged K48 ubiquitin, HA-tagged K63 ubiquitin, HA-tagged K48R ubiquitin and HA-tagged K63 ubiquitin. j, Immunoblot analysis of TRAF6 ubiquitination of LPS-stimulated macrophages transfected with Control siRNA or SLAMF7 siRNA, followed by LPS stimulation for 30 min plus MG132 treatment or not. k, Relative expression of Tnf, $\|1 \mathrm{~b}\|$,6 and $\| 10$ after transfected with constructed TRAF6 plasmid and Control plasmid. Data are the mean \pm SEM and represent three individual experiments. ns, not significant; ${ }^{*} \mathrm{P}<$ $0.05 ; * \star, P<0.01 ; * \star *, P<0.001$. 
a

\section{SLAMF7 function domain:}

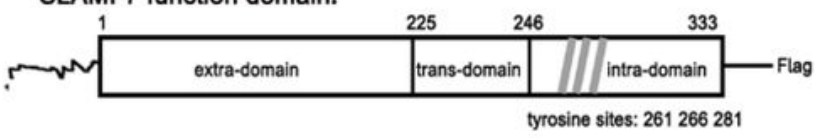

b

SHIP1 function domain:

\begin{tabular}{|c|c|c|c|}
\hline SH2 & & EEP & P rich \\
\hline
\end{tabular}

C

\begin{tabular}{|c|c|c|c|}
\hline SHIP1-HA & + & + & + \\
\hline SLAMF7-Flag & + & - & 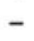 \\
\hline$\triangle$ intra-Flag & - & + & - \\
\hline$\Delta$ trans-Flag & - & - & + \\
\hline$\Delta$ extra-Flas & - & - & - \\
\hline
\end{tabular}

IP: Flag $\mid \begin{aligned} & \text { IB: HA } \\ & \text { IB: Flag }\end{aligned}$

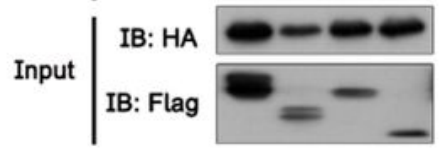

d
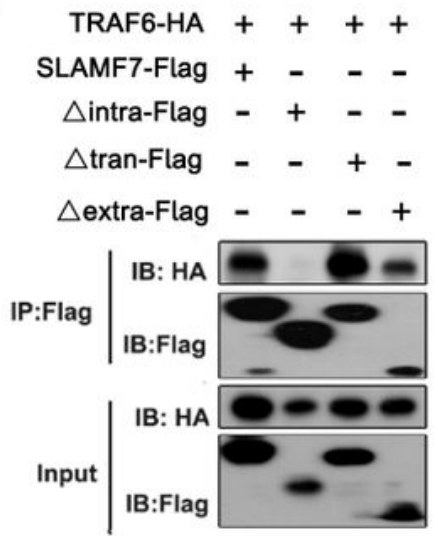

e

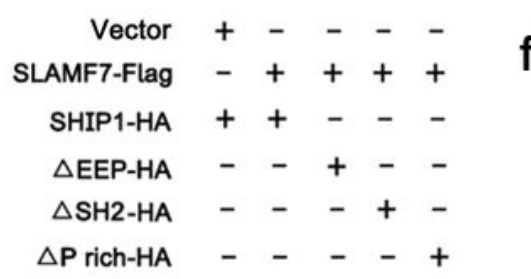

f

TRAF6-Flag ++++
SHIP1-HA +---
$\triangle$ SH2-HA -+--
$\triangle$ EEP-HA -+-
$\triangle$ P rich-HA $--\quad+$

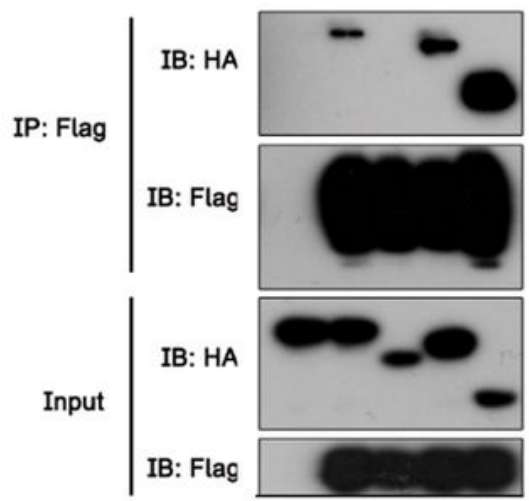

g

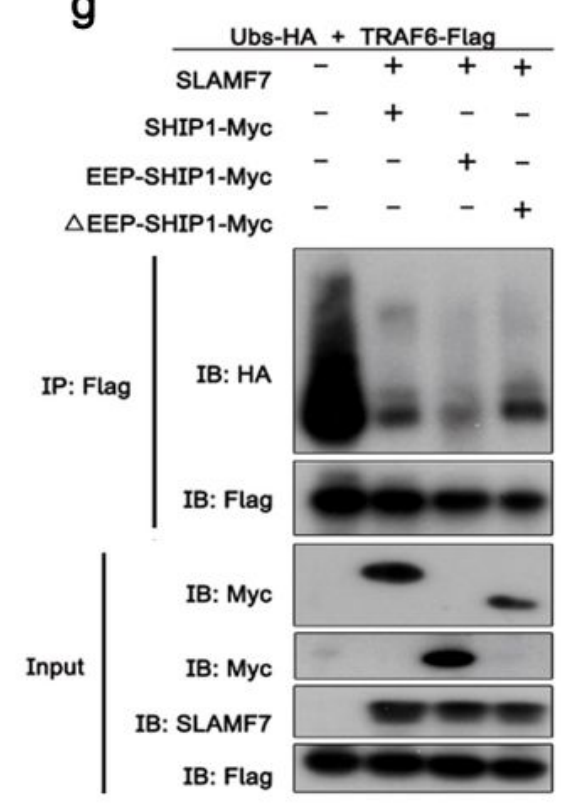

h

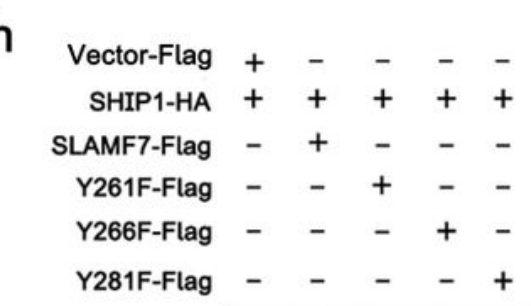

i

$\begin{array}{rlllll}\text { Vector-Flag } & + & - & - & - & - \\ \text { TRAF6-HA } & + & + & + & + \\ \text { SLAMF7-Flag } & - & + & - & - & - \\ \text { Y261F-Flag }- & - & + & - & - \\ \text { Y266F-Flag } & - & - & - & + & - \\ \text { Y281F-Flag } & - & - & - & - & +\end{array}$
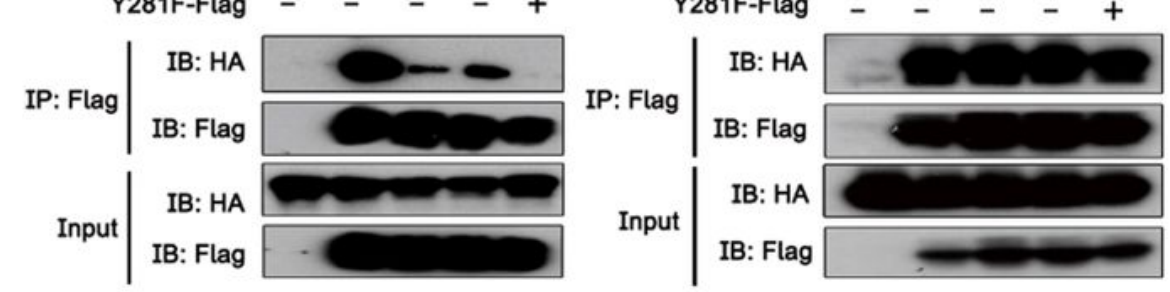

\section{Figure 5}

Binding of SLAMF7 to SHIP1 by tyrosine and EEP domain is required for negative regulation of TLR responses. a, Flag-tagged SLAMF7 plasmids lacking extracellular ( $\triangle$ extra from 225 aa to 333 aa), lacking transmembrane ( $\triangle$ trans from 1 aa to 224 aa plus 246 to 333 aa) or lacking intracellular ( $\triangle$ intra from 1 aa to 245 aa) domains evolved from whole sequence of SLAMF7. The tyrosine sites at 261, 266 and 281 aa of SLAMF7 amino sequence, ignoring signal peptide manually mutated to phenylalanine 
$(\mathrm{Y} \rightarrow \mathrm{F})$, respectively. b, Analysis function domains of SHIP1 and SLAMF7 amino sequences. Truncated SHIP1 plasmids consisted of HA-tagged SH2 domain (SH2-HA, from 1 aa to 105 aa), HA-tagged EEP domain (EEP-HA, from 407 aa to 713 aa) and HA-tagged $P$ rich domain (P rich-HA, from 714 aa to 1191 aa) as well as HA-tagged SHIP1 lacking SH2 domain ( $\triangle \mathrm{SH} 2-\mathrm{HA})$, SHIP1 lacking EEP domain $(\triangle \mathrm{EEP}-\mathrm{HA})$ and SHIP1 lacking $P$ rich domain ( $\triangle \mathrm{P}$ rich-HA). c, Immunoprecipitation using anti-Flag or anti-HA antibodies from lysates of 293T cells transfected with Flag-tagged SLAMF7 function domains as well as HA-tagged SHIP1. d, Immunoprecipitation using anti-Flag antibodies from lysates of 293T cells transfected with Flag-tagged SLAMF7 function domains as well as HA-tagged TRAF6. e, Immunoblot analysis of anti-HA from the precipitation lysate by using anti-Flag from 293T cells co-transfected with distinct HA-tagged SHIP1 plasmids mentioned above and Flag-tagged SLAMF7. f, Immunoprecipitation using anti-Flag antibodies from lysates of 293T cells co-transfected with distinct HA-tagged SHIP1 plasmids mentioned above and Flag-tagged TRAF6. $\mathrm{g}$, Immunoblot assay of endogenous ubiquitination of TRAF6 by using anti-HA from lysate immunoprecipitation with anti-Flag from 293T transfected with HA-tagged ubiquitin and Flag-TRAF6, plus SLAMF7 alone or SLAMF7 and EEP-HA, SLAMF7 and $\triangle E E P-$ HA. h, i, Immunoblot assay of anti-HA and anti-Flag of lysate precipitation by using anti-Flag antibody from 293T cells transfected with HA-tagged SHIP1 and Flag-tagged SLAMF7 tyrosine mutation (h), or HAtagged TRAF6 and Flag-tagged SLAMF7 tyrosine mutation (i). j, Tnf, $\| 1 \mathrm{~b}$ and $\| 6$ mRNA levels in RAW264.7 cells transfected with Y261F, Y266F or Y281F-SLAMF7 plasmid compared with vector, followed by LPS stimulation for $6 \mathrm{~h}$. Data are the mean \pm SEM and represent three individual experiments. ns, not significant; ${ }^{*}, \mathrm{P}<0.05 ;{ }^{* \star}, \mathrm{P}<0.01$; ${ }^{\star \star \star}, \mathrm{P}<0.001$. 
a

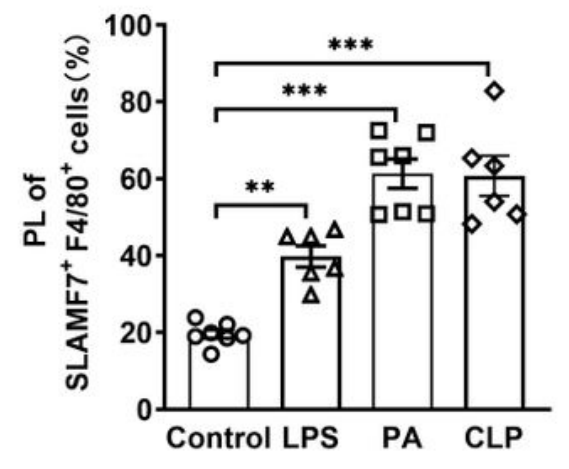

d

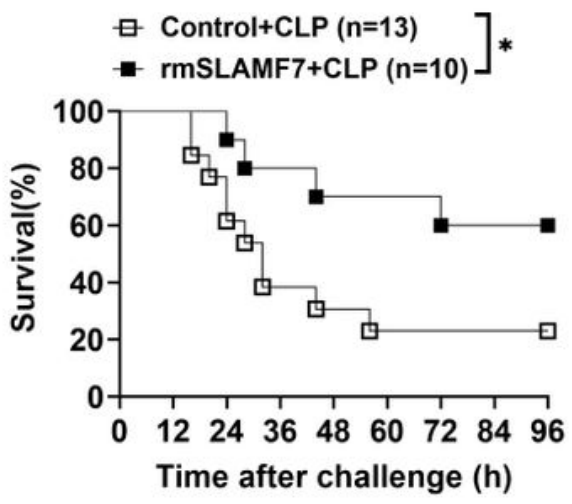

f

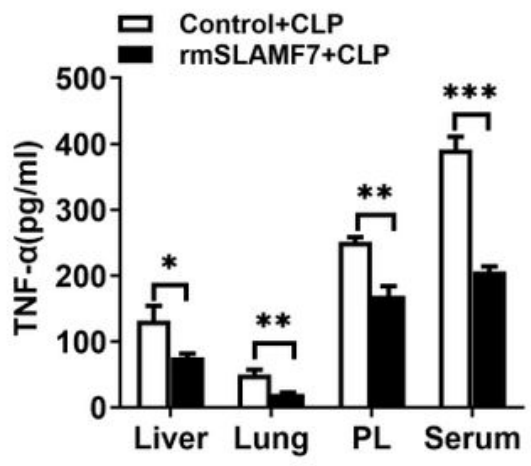

b

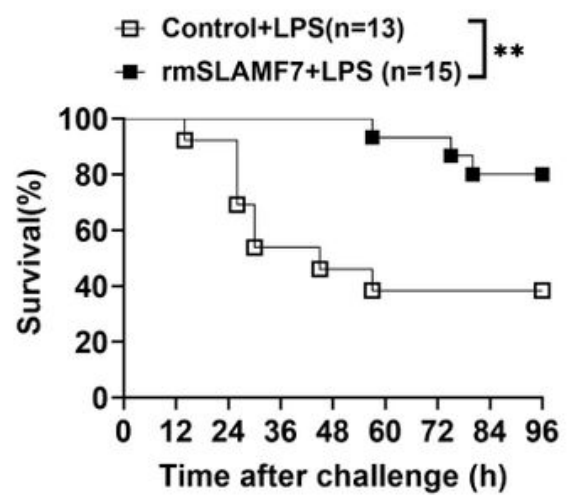

e

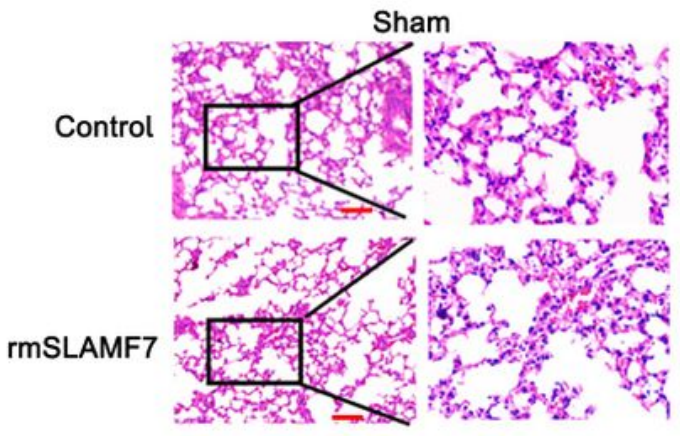

C

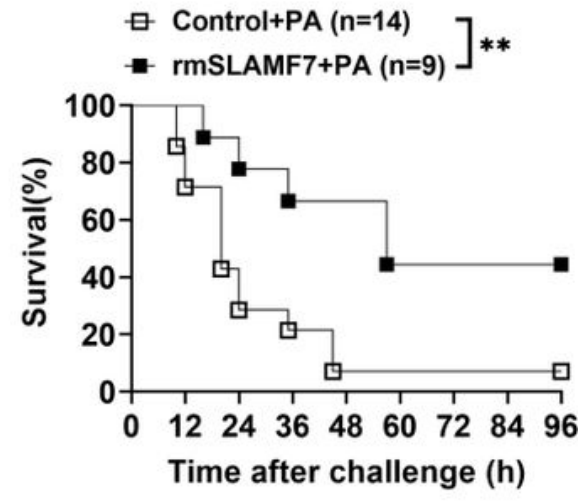

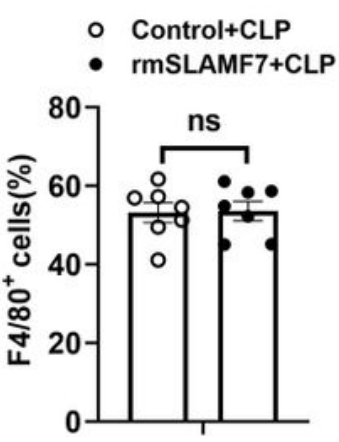
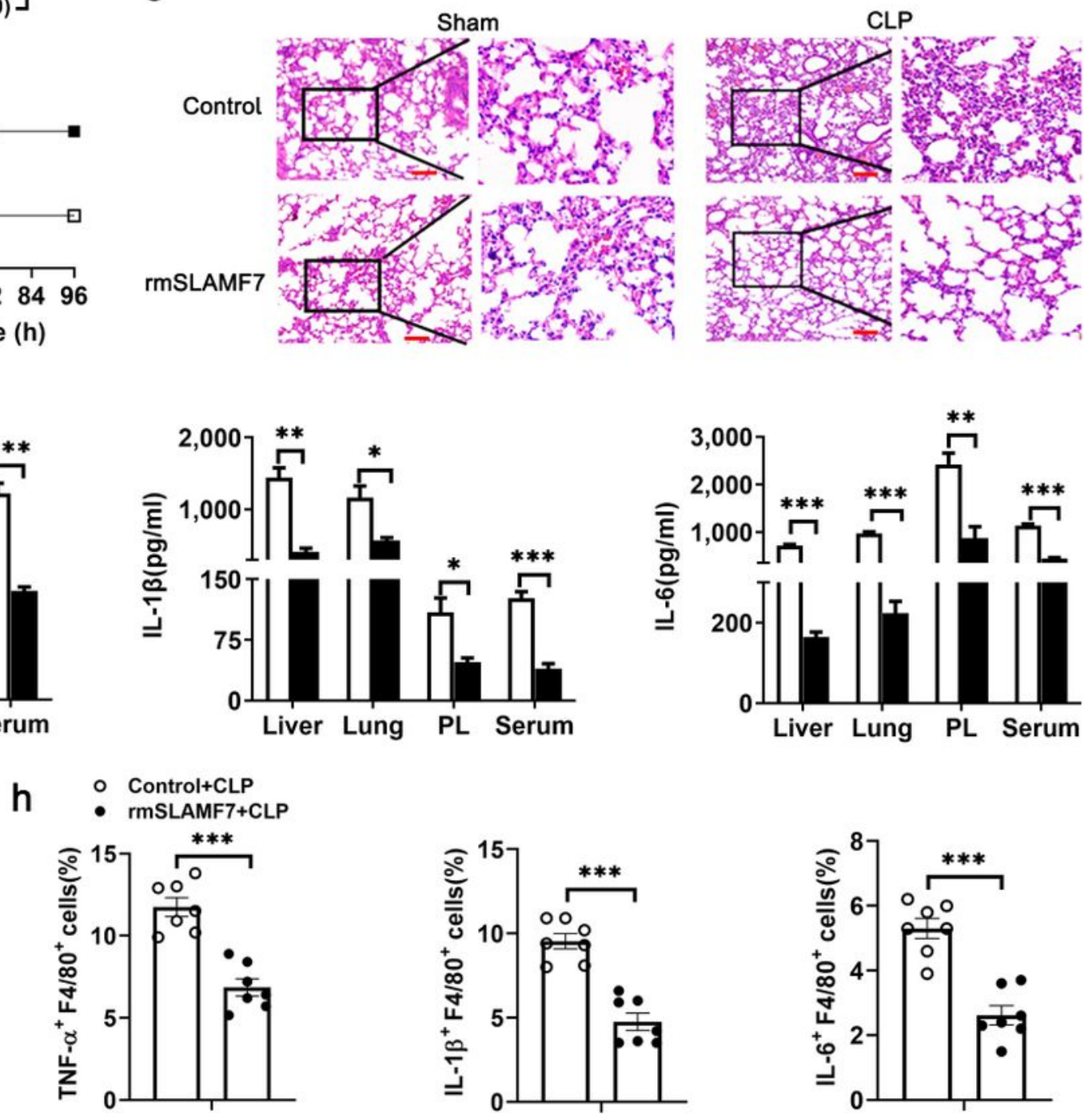

\section{Figure 6}

SLAMF7 protects against sepsis by inhibiting pro-inflammatory cytokines production a, Septic murine models were established by intraperitoneal (i.p.) injection with LPS, PA or CLP surgery. The percentage of SLAMF7 expressed on macrophages isolated from peritoneal lavage (PL) of Wild-type (WT) C57BL/6 (B6) mice was analyzed by flow cytometry. b-d, Survival rate of WT mice challenged with LPS (b), PA (c) or CLP (d) for $2 \mathrm{~h}$, followed by intraperitoneal injection with rmSLAMF7 vs vehicle control $(0.9 \% \mathrm{NaCl})$. e, 
Hematoxylin-and-eosin staining of lung sections was examined in rmSLAMF7 vs vehicle control-treated WT mice at $12 \mathrm{~h}$ after CLP. Scale bars, $20 \mu \mathrm{m}$. $\mathrm{f}$, The protein levels of TNF-a, IL-1 $\beta$ and IL- 6 in the supernatant of Liver, Lung, peritoneal lavage (PL) and blood (serum) from mice pre-challenged with CLP, followed by injection with rmSLAMF7 for $24 \mathrm{~h}$ later. $\mathrm{g}$, The percentage of F4/80+cells in PL from mice pre-challenged with CLP, followed by injection with rmSLAMF7 or control $(0.9 \% \mathrm{NaCl})$ for $24 \mathrm{~h}$ later. $\mathrm{h}$, The percentage of TNF-a+, IL-1 $\beta+$ and IL-6+ F4/80+ cells from PL were analyzed by flow cytometry for each group. Data are the mean \pm SEM and represent three individual experiments. ns, not significant; *, $\mathrm{P}<$ $0.05 ; * \star, P<0.01 ; * \star \star, P<0.001$. 
a

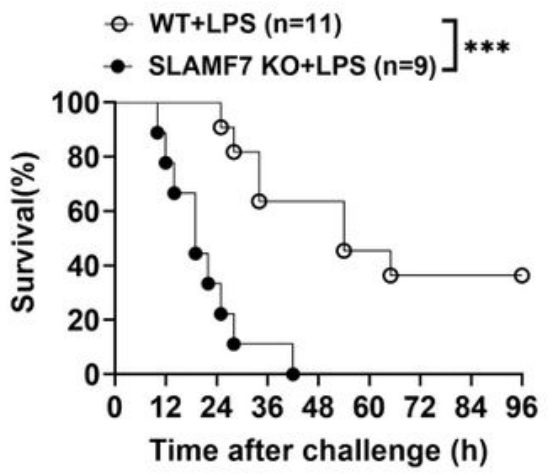

d

SLAMF7 KO

WT

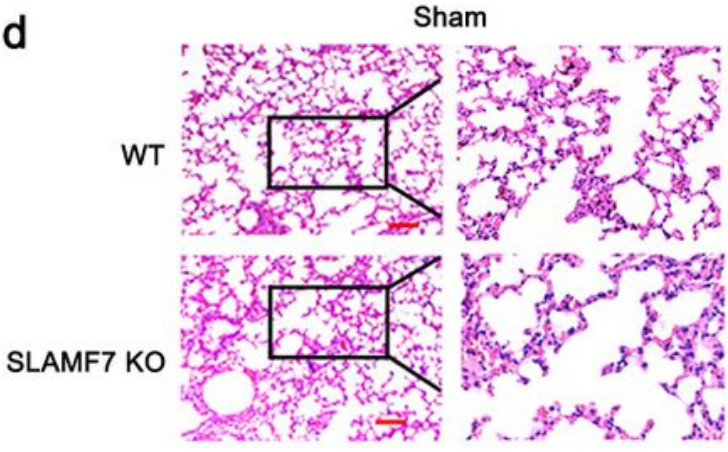

b

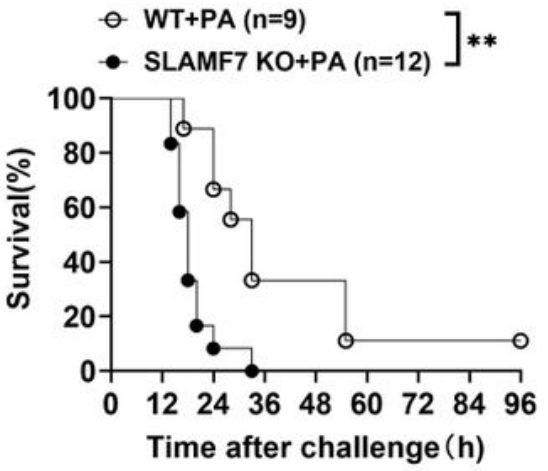

C

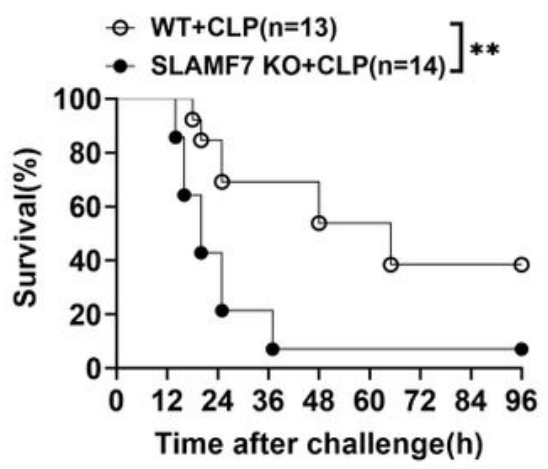

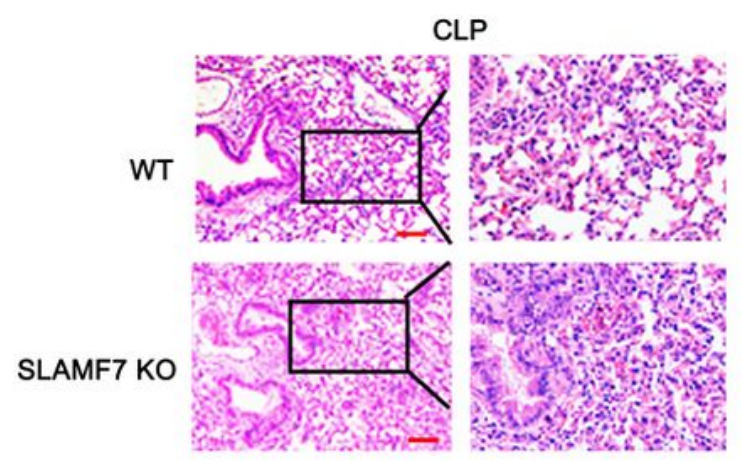

e
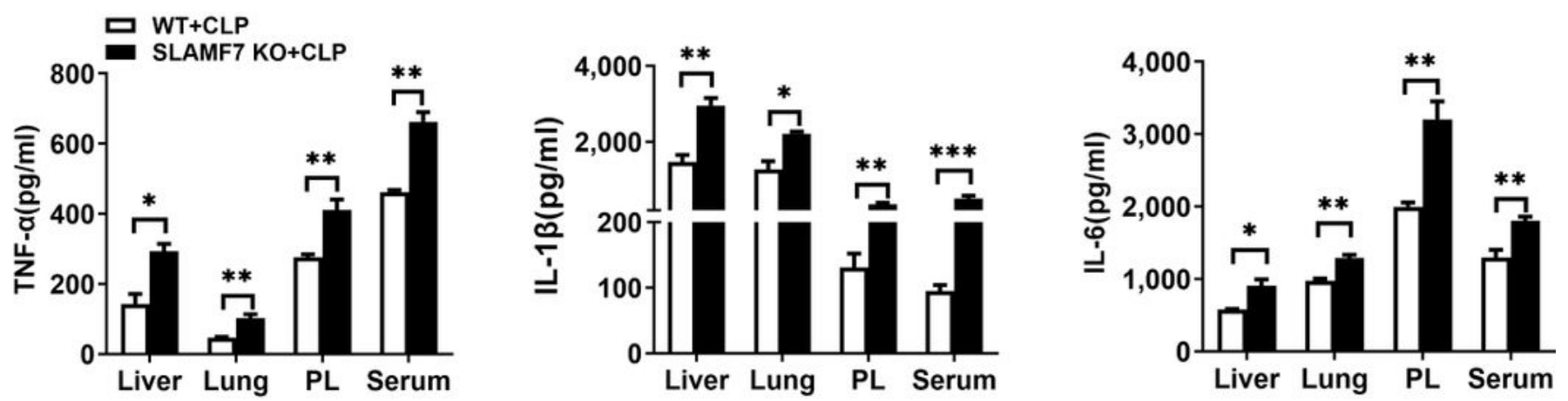

- WT CLP

- SLAMF7 KO CLP

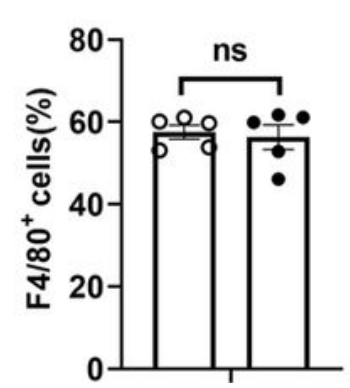

g

- WT CLP

- SLAMF7 KO CLP

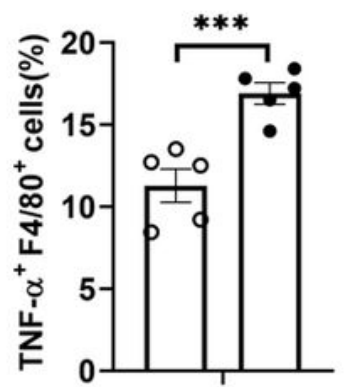

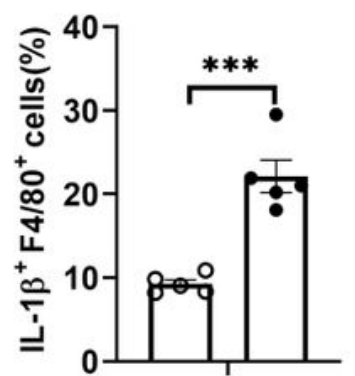

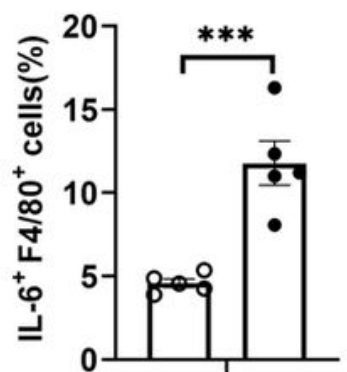

Figure 7

SLAMF7 deficiency promoted disease severity of sepsis. a-c, Survival rate of SLAMF7 KO vs WT mice challenged with LPS (a), PA (b) or CLP (c). d, Hematoxylin-and-eosin staining of lung sections was

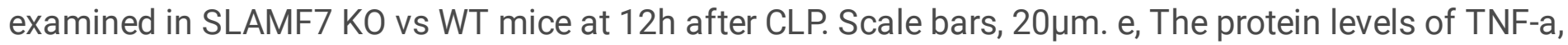
IL-1 $\beta$ and IL- 6 in the supernatant of Liver, Lung peritoneal lavage (PL) and blood (serum) from WT and SLAMF7 KO mice 24h after CLP. f, The percentage of F4/80+ cells in PL from WT B6 and SLAMF7 KO 
mice after CLP for $24 \mathrm{~h}$ were analyzed by flow cytometry. $\mathrm{f}$, The percentage of TNF-a+, IL-1 $\beta+$ and IL-6+ $\mathrm{F} 4 / 80+$ cells from PL were analyzed by flow cytometry for each group. Data are the mean \pm SEM and represent three individual experiments. ns, not significant; *, $P<0.05 ; * \star, P<0.01$; ***, $P<0.001$.

\section{Supplementary Files}

This is a list of supplementary files associated with this preprint. Click to download.

- SLAMF7ANDSEPSISSupplementary.pdf 